\title{
AZT acts as an anti-influenza nucleotide triphosphate targeting the catalytic site of A/PR/8/34/H1N1 RNA dependent RNA polymerase
}

\author{
Nataraj Sekhar Pagadala ${ }^{1,2,3,4}$ iD
}

Received: 31 October 2018 / Accepted: 2 February 2019 / Published online: 9 February 2019

(c) Springer Nature Switzerland AG 2019

\begin{abstract}
To develop potent drugs that inhibit the activity of influenza virus RNA dependent RNA polymerase (RdRp), a set of compounds favipiravir, T-705, T-1105 and T-1106, ribavirin, ribavirin triphosphate viramidine, 2FdGTP (2'-deoxy-2'fluoroguanosine triphosphate) and AZT-TP (3'-Azido-3'-deoxy-thymidine-5'-triphosphate) were docked with a homology model of IAV RdRp from the A/PR/8/34/H1N1 strain. These compounds bind to four pockets A-D of the IAV RdRp with different mechanism of action. In addition, AZT-TP also binds to the PB1 catalytic site near to the tip of the priming loop with a highest $\Delta \mathrm{G}$ of $-16.7 \mathrm{Kcal} / \mathrm{mol}$ exhibiting an $\mathrm{IC}_{50}$ of $1.12 \mu \mathrm{M}$ in an in vitro enzyme transcription assay. This shows that AZT-TP mainly prevents the incorporation of incoming nucleotide involved in initiation of vRNA replication. Conversely, 2FdGTP used as a positive control binds to pocket-B at the end of tunnel-II with a highest $\Delta \mathrm{G}$ of $-16.3 \mathrm{Kcal} / \mathrm{mol}$ inhibiting chain termination with a similar $\mathrm{IC}_{50}$ of $1.12 \mu \mathrm{M}$. Overall, our computational results in correlation with experimental studies gives information for the first time about the binding modes of the known influenza antiviral compounds in different models of vRNA replication by IAV RdRp. This in turn gives new structural insights for the development of new therapeutics exhibiting high specificity to the PB1 catalytic site of influenza A viruses.
\end{abstract}

Keywords RNA dependent RNA polymerase $\cdot$ Catalytic site $\cdot$ Docking $\cdot$ Nucleotide triphosphates

\section{Introduction}

Influenza is a highly contagious airborne viral infection with the potential to cause seasonal outbreaks ranging from mild fatigue to respiratory illness and death. The processes of viral replication and transcription is controlled by its RNA dependent-RNA polymerase which is a heterotrimeric

Electronic supplementary material The online version of this article (https://doi.org/10.1007/s10822-019-00189-w) contains supplementary material, which is available to authorized users.

Nataraj Sekhar Pagadala

nattu251@gmail.com

1 Li Ka Shing Institute of Virology, University of Alberta, Edmonton, AB, Canada

2 Li Ka Shing Applied Virology Institute, University of Alberta, Edmonton, AB, Canada

3 Department of Medical Microbiology and Immunology, University of Alberta, Edmonton, AB, Canada

4 Medical Microbiology and Immunology, Li Ka Shing Institute of Virology, University of Alberta, Edmonton, AB T6G 2E1, Canada protein containing three subunit proteins: polymerase basic 1 (PB1), polymerase basic2 (PB2), and polymerase acidic (PA) proteins. Both $\mathrm{PB} 1$ and $\mathrm{PB} 2$ appear in all influenza viruses, whereas the third subunit, polymerase acidic (PA) occurs only in influenza A and B viruses, while polymerase 3 subunit (P3) is seen only in influenza $C$ viruses [1,2]. The catalytic site of the IAV RdRp resides at the edge of a large central cavity formed by PB1 and the N terminus of PB2 [3-5]. The nitrogenous base of the first NTP during de novo initiation is stabilized by the priming loop (a $\beta$-hairpin structure) of the PB1 thumb domain that protrudes into the central cavity of the active site [5-8]. Recently, the importance of the priming loop in the initiation of primer-independent replication on the vRNA template was clearly shown $[9,10]$. Due to its high conservation among different strains, IAV RdRp contains multiple sites for potential antiviral drug development. Using this information, different classes of drugs were developed including nucleoside analogs such as favipiravir (T-705), peramivir and 2'-deoxy-2'-fluoroguanosine, pyrazine carboxamide derivatives T-705 and T-1106, ribavirin and its amidine prodrug viramidine, and $2^{\prime} \mathrm{F}-2^{\prime} \mathrm{dNTP}$ that inhibits IAV RNA synthesis chain elongation [11-15]. In 
addition, different types of phytochemicals, marchantins, plagiochin A and perrottetin F were developed to inhibit the endonuclease activity of IAV RdRp. Furthermore, a large number of cap-snatching inhibitors have been reported targeting the endonuclease site of IAV RdRp such as 4-substituted 2,4-dioxobutanoic acid derivatives, diketo compounds and green tea catechin, $N$-hydroxamic acid/ $N$-hydroxyimide and JNJ-872 that bind to the viral endonuclease and inhibits the cleavage of mRNA 11-13 nucleotides downstream of the cap [16-20]. Recently, the therauptic agent Baloxavir marboxil approved by FDA under the trade name Xofluza was developed by Shionogi Co to treat uncomplicated influenza viral infection for the patients under 12 years of age or older with less than $48 \mathrm{~h}$ of infection [21]. Furthermore, both transcriptase and endonuclease inhibitors such as Flutamide and 3-substituted 2,4-dioxobutanoic acids were identified to inhibit the cap-dependent endonuclease activity of RdRp [16]. Recently, several inhibitors that bind to the PA endonuclease domain were developed such as S-033188 and AL-794 (https://clinicaltrials.gov/ct2/show/NCT02588521), cycloheptathiophene-3-carboxamide, polyamido derivatives, AL18, benzbromarone, diclazuril, Benzofurazan-derivatives and licorice-derived compounds that inhibits IAV RdRp binding to the PA (C) subunit [22-30]. Finally, siRNA in combination with an RNA polymerase inhibitor suggested that the PA subunit of RNA polymerase is a promising target for anti-influenza virus agents [31]. Apart from small molecules, a 15-mer phosphorothioate oligonucleotide derived from the 5'-end of the PB2 segment-1 was developed as an inhibitor [32]. Subsequently, 5'-capped short phosphorothioate RNA fragments and cap decoys synthesized by T7 RNA polymerase in vitro were developed to inhibit RNA polymerase gene expression [33]. Similarly, short capped oligonucleotides were synthesized to inhibit cap-primed transcription at the initial step of cap binding and ApGprimed viral transcription [34]. Although many drugs were proven experimentally both in vitro and in vivo, no approved drug targeting the catalytic site of this enzyme is currently available for human use.

In the present study, the three-dimensional structure of IAV RdRp from the A/PR/8/34/H1N1 strain was developed by homology modelling and its mechanism of vRNA replication was studied using the extended primer from $\Phi 6$ polymerase and the elongation template from polio virus. Further, a set of known anti-influenza nucleotide triphosphates that are proven both in vitro and in vivo were successfully docked against the built homology model in different models I-IV (Fig. S1). To prove the accuracy of docking and their mode of binding, AZT-TP that was used as an antiviral compound for HIV was docked to the same homology model and were then confirmed as inhibitor in purified IAV polymerase assay from strain A/PR/8/34/H1N1. To further prove the effect of AZT-TP inhibition compared to known inhibitors, 2FdGTP that was proven as IAV RdRp inhibitor was used as a positive control. Finally, based on the correlation between the docking and previous experimental studies, our model clearly differentiated IAV RdRp drugs using the structural features associated with the chemical modifications of the compounds which provides new insights to develop effective influenza antivirals.

\section{Methodology}

\section{Homology modelling and docking}

The initial homology model of A/PR/8/34/H1N1 RdRp was built with the template structure of bat polymerase (PDB: 4WSB) using the software MODELLER 9v7 on windows operating system [35]. The query sequences from A/ $\mathrm{PR} / 8 / 34 / \mathrm{H} 1 \mathrm{~N} 1$ strain was searched to find out the related protein structure to be used as a template by the BLAST (Basic Local Alignment Search Tool) program against PDB (Protein Databank) [36]. Sequences of PA, PB1 and PB2 showed maximum identity of 70\%, 79\% and 68\% with high score and less e-value with the reference structure to build a 3D model for A/PR/8/34_H1N1 polymerase. The domain sequences of PA, PB1 and PB2 (Accession Numbers: ABO21708.1, ABO21705.1 \& ABD77683.1) of A/ PR/8/34_H1N1 polymerase was obtained from NCBI. The co-ordinates for the structurally conserved regions (SCRs) for A/PR/8/34_H1N1 sequences were assigned from the template using pair wise sequence alignment, based on the Needleman-Wunsch algorithm [37, 38]. The 3D model obtained was evaluated for its stereo chemical quality by Ramachandran's map using PROCHECK (Programs to Check the Stereo Chemical Quality of Protein Structures), ERRAT and verify_3D programs [39]. The residue packing and atomic contact analysis was performed by using the Whatif program to identify bad packing of side chain atoms or unusual residue contacts. Secondary structures of proteins were analyzed and compared by the SPDBV (Swiss Protein Databank Viewer) software [40]. The Site Finder module of the MOE was used to identify possible substratebinding pockets within the homology model of IAV RdRp [41-43]. Further, known IAV RdRp nucleotide triphosphates and AZT-TP were used for molecular docking against the homology model of A/PR/8/34/H1N1 polymerase in four different models (1) In the presence of influenzaA vRNA, (2) In the presence of capped primer mapped on to homology model of A/PR/8/34/H1N1 from $\phi 6$ polymerase, (3) Influenza A vRNA is replaced with influenza B vRNA and IV. In the presence of influenzaA vRNA with extended primer and 3 '-elongation template. The partial charges for these models were assigned using Tripos Assisted Force Field (TAFF) parameterized for small organic molecules. Simultaneously, 
hydrogens and lone pairs were adjusted using the same forcefield at $\mathrm{pH}$ 7. The ligand was optimized by energy minimization with the same TAFF Force Field until the energy gradient is below $0.001 \mathrm{Kcal} / \mathrm{Mol}$ [44]. In silico docking in MOE (Chemical computing group, Montreal, Canada), the ligand placement method "Alpha PMI" was used to generate 1000 docking poses in each case. The top best solution based upon the London $\mathrm{dG}$ scoring function using implicit generalized born solvation model were further refined using the TAFF forcefield calculations and subject to rescoring using the same scoring function described above [45]. The simulated annealing based on the Monte Carlo method was used to find the global minimum of the ligand [46]. Finally, affinity scoring function, $\Delta \mathrm{G}$ (Utotal in $\mathrm{kcal} / \mathrm{mol})$, was employed to rank a candidate poses as the sum of the electrostatic and Van der Waals energies. The dielectric function based on the reaction model with a cut-off between 8 and $10 \AA$ was used for our docking studies with the pocket radius of $6 \AA$. All the solvent water was included during the alpha site finding and docking calculations. By default, thirty conformations docked as a cut-off were retained. Pharmacophore analysis was also predicted using MOE software which is a set of structural features in a ligand that are directly related to the ligand's recognition at a receptor site and its biological activity.

\section{Experimental study}

The effect of AZT-TP and 2'-deoxy-2'-fluoroguanosine triphosphate that binds and inhibits the catalytic activity of RdRp inhibiting the incorporation of GTP into TCA-precipitable material was investigated using the A/PR/8/34 (PR8) strain (Table 1). Further, purified influenza Virus A/PR/8/34/ H1N1 that was obtained from Charles river (MD) was disrupted with $2.5 \%$ Triton $\mathrm{N}-101$ and diluted $1: 2$ with $0.25 \%$
Triton N-101. Disruption provided the source of influenza ribonucleoprotein (RNP) containing the IAV RdRp and template vRNA. Samples were stored on ice until use in the assay. Six serial half-log dilutions of the test drugs (twelve drugs) at concentrations of $0.001,0.01,0.1,1,10$ and $100 \mu \mathrm{M}$ and positive control 2 '-deoxy-2'-fluoroguanosine 5 '-triphosphate (high tests of $100 \mu \mathrm{M}$ ) were tested in triplicate. Each polymerase reaction contained the following: disrupted RNP, Tris-HCL, KCL, MgCl2, dithiothreitol, $0.25 \%$ Triton N-101, $\left[\alpha-{ }^{32} \mathrm{P}\right]$ GTP, ATP, CTP, UTP, GTP, and adenyl $\left(3^{\prime}-5^{\prime}\right)$ guanosine. For testing the inhibitor, the reactions contained the inhibitor and the same was done for reactions containing the positive control. The reaction was incubated at $30^{\circ} \mathrm{C}$ for $1 \mathrm{~h}$, transferred onto glass-fiber filter plates and precipitated with trichloroacetic acid (TCA). Once the filter has dried, incorporation of $\left[\alpha-{ }^{32} \mathrm{P}\right]$ GTP was measured using a scintillation counter (Microbeta). Negative control reactions were prepared by omitting RNP complexes whereas positive control reactions for polymerase inhibitors contained the specific inhibitor 2'-deoxy-2'-fluoroguanosine 5 '-triphosphate (2FdGTP). Inhibition of viral polymerase activity was measured by $\mathrm{IC}_{25}, \mathrm{IC}_{50}$ and $\mathrm{IC}_{95}$. Finally, the docking conformations of these polymerase inhibitors were defined by the dihedral angles $\Phi_{\mathrm{T}} \mathrm{C}\left(2^{\prime}\right)$ - $\mathrm{C}\left(1^{\prime}\right)-\mathrm{N}(1)$ $\mathrm{N}(5) / \mathrm{C}(6)$ and $\Phi_{\mathrm{P}} \mathrm{O}\left(5^{\prime}\right)-\mathrm{C}\left(1^{\prime}\right)-\mathrm{N}(1)-\mathrm{N}(5) / \mathrm{C}(6)$ (Fig. S2).

\section{Results}

\section{Homology model of A/PR/8/34/H1N1 RdRp}

For the purpose of this study, a full complex structure of the IAV RdRp from A/PR/8/34/H1N1 strain was built using homology modeling. Of the twenty structures calculated for the same target (A/PR/8/34/H1N1 RdRp) using the template

Table 1 Residues lining the three tunnels of A/PR/8/34/H1N1 RdRp

\begin{tabular}{|c|c|c|c|}
\hline Tunnel & PA & PB1 & PB2 \\
\hline 1 & $\begin{array}{l}\mathrm{Arg}^{512}, \mathrm{Asn}^{513} \\
\mathrm{Asp}^{514}, \mathrm{Thr}^{515} \\
\text { and } \mathrm{Asp}^{516}\end{array}$ & $\begin{array}{l}\operatorname{Arg}^{126}, \operatorname{Gln}^{127}, \operatorname{Leu}^{133}, \operatorname{Asn}^{134}, \operatorname{Arg}^{135}, \operatorname{Asn}^{136}, \operatorname{Gln}^{137}, \\
\operatorname{Met}^{356}, \operatorname{Tyr}^{355}, \operatorname{Ser}^{673}, \operatorname{Ile}^{674}, \operatorname{Asn}^{676} \text { and Gln }\end{array}$ & \\
\hline 2 & & 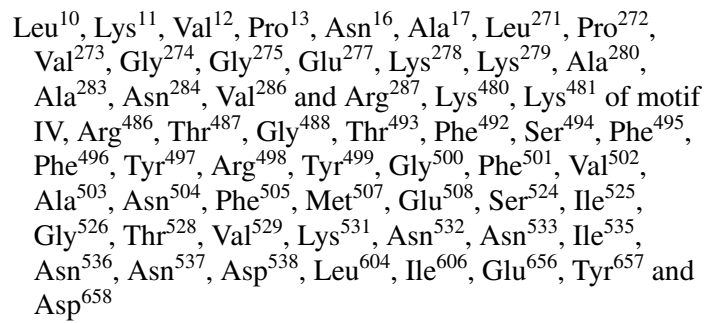 & 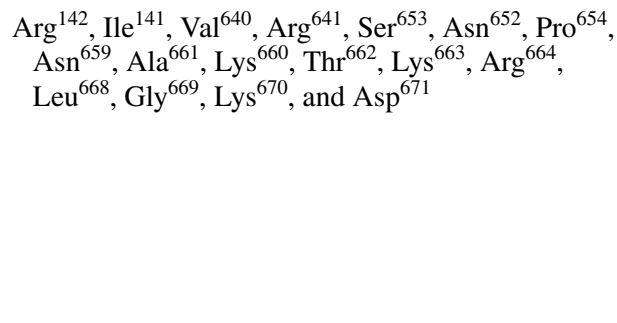 \\
\hline 3 & $\begin{array}{l}\text { Gly }^{657}, \text { Phe }^{658} \\
\text { Alal }^{660}, \text { Glu }^{661} \\
\text { Glu }^{688} \text { and } \\
\text { Ala }^{689}\end{array}$ & $\begin{array}{l}\operatorname{Asn}^{306} \text { (Motif-I), Thr } \\
\text { Asp }^{346} \text { (Motif-III) }\end{array}$ & \\
\hline
\end{tabular}


(4WSB), with MODELLER 9v7 software, the one with the lowest energy (MODELLER objective function) which was predicted to be stable in the biological system was selected as the best model for A/PR/8/34/H1N1. The geometry of the final refined model evaluated with Ramachandran's plot calculations computed with the PROCHECK program reveals that $93.7 \%, 93.1 \%$ and $92.9 \%$ of the residue's backbone $\varphi$ and $\phi$ dihedral angles of PA, PB1 and PB2 domains of IAV RdRp falls within the most favored and allowed regions of the Ramachandran's plot. The overall PROCHECK G-factor of $-0.16,-0.17$ and -0.13 and quality factor of 63.4 , 71.5 and 70.5 in the ERRAT graphs for PA, PB1 and PB2 domains indicate acceptable protein environments with the environment profiles of VERIFY_3D above zero. Further evaluation of the structural integrity of the final model of IAV RdRp using the Prosa web server showed a $\mathrm{Z}$ score of -16.5 making from which we conclude that the refined model was very near to the crystal structure value of -16.63 and therefore is highly reliable. The structure of A/PR/8/34/ H1N1 RdRp begins with $\mathrm{Asn}^{53}$ and $\mathrm{Ser}^{8}$ indicating that the $\mathrm{N}$-terminal region from $\mathrm{Met}^{1}-\mathrm{Arg}^{52}$ is highly exposed and susceptible to proteolytic cleavage. The $\mathrm{RdRp}$ model is mainly composed of three structural domains PA, PB1 and PB2. The PB2 domain is again subdivided into three sub domains comprising the fingers, palm and thumb adopting a typical right-handed RdRp structure. The $0.8 \AA$ RMSD of the PB1 domain showed that the folds of finger, palm and thumb are similar to the template bat polymerase. The finger subdomain and the thumb domain are connected to each other with the linker that protrudes from the fingertips. The priming loop (residues Asn ${ }^{641}-\mathrm{Asp}^{658}$ ) of PB1 contains the highly conserved motif 648-AHGP at the loop tip which points from the palm subdomain towards the active site of palm. The two tunnels were observed that are placed below and above the priming loop. The first tunnel was observed below the priming loop between the fingers and the thumb and the second tunnel which is roughly perpendicular to the first goes across the entire protein to allow exit of the viral RNA template (Fig. 1). The total length of the two tunnels starting from the PA promoter site to the end of the exit channel is approximately $32 \AA$ that was not shown previously. The residues involved in the formation of both tunnels and their motifs that are important for catalytic activity are shown in Table 1 and Fig. 2. The residues of motif I and motif III from PB1 and from the PA domain help in the formation of the third tunnel for the incoming passage of NTPs near to the priming loop as show in Table 1 and Fig. 2.

Interactions of the vRNA template promoter with $\mathrm{A} /$ PR/8/34/H1N1 RdRp shows that the cavity formed by both PA_C and PB1 subunits are involved in binding the 3 '-nucleotides 6-9 of the purine rich 5'-activator, whereas nucleotides 1-5 only interact with PB1 of the 5 '-activator (Fig. 3). Residues 670-679 of PB1 are involved in binding the single

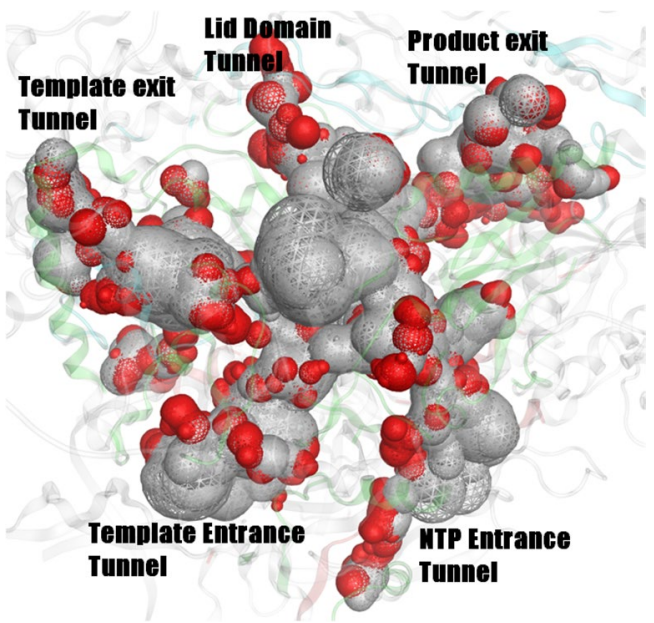

Fig. 1 Internal tunnels predicted for the homology model of IAV RdRp from A/PR/8/34/H1N1 strain using MOE software suite. The template entrance and exit, NTP entrance and product exit are indicated in surface representation. Both charged and hydrophobic surfaces are indicated in red and grey colours respectively

stranded 3 '-extremity, U9, U10 and U12 of the 5'-activator. In addition, the residues from the PB1-ribbon $\mathrm{Lys}^{188}(3.1 \AA)$ and $\operatorname{Thr}^{201}$ (2.6 $\AA$ ) interacts with the proximal $3^{\prime}$-nucleotide G2. Furthermore, PB2 residues $\operatorname{Ser}^{36}$ (3.3 $\AA$ ) and $\mathrm{Arg}^{38}$ (3.1 $\AA$ ) are involved in binding to U12 of the single stranded 3 '-extremity. The PA domain from $\mathrm{Arg}^{508}-\mathrm{Phe}^{520}$ forms a wedge that separates the $5^{\prime}$ - and 3 '-strands into the binding pockets that create contacts between $\mathrm{Arg}^{512}$ and $3^{\prime}$-single strand bases U9 (3.6 $\AA$ ) and U12 (3.1 $\AA$ ) of the template strand of the vRNA. In turn, $\mathrm{Arg}^{508}$ and $\mathrm{Val}^{517}$ of the beta sheet shows contacts with the 3'A6 phosphate backbone (4.1 $\AA$ ) and the $3^{\prime}-\mathrm{C} 7$ of the side chain (3.4 $\AA$ ). The positively charged residues in the semicircular groove of PA_C, namely $\mathrm{Arg}^{566}$ and lys ${ }^{572}$ of the antiparallel beta sheet shows contact with $\mathrm{C} 11$ and U14 of both strands of the vRNA. However, $\operatorname{Arg}^{279}(3.5 \AA), \operatorname{Lys}^{281}(3.3 \AA), \mathrm{Lys}^{328}$ (2.8 and $3.3 \AA$ ) and Lys ${ }^{539}$ (3.9) of PA_C shows contacts with U14 and A16 of the template 3'-strand of the vRNA. Furthermore, the arch motif of PA_C domain comprising 367-LysTrp-Ala-Leu-Gly-Glu-Asn-Met-Ala-Pro-Glu-Lys-Val-379 forms a binding loop that shows contacts with the phosphate backbone of A6 and A7 of the template vRNA. Replacing the vRNA promoter of influenza A with influenza B promoter allows the 3'-end of nucleotides U13-C17 to interact with PB1 in the narrow template entrance tunnel. The residues $\mathrm{Asn}^{225}$ and $\mathrm{Gln}^{127}$ of PB1 are involved in binding the single stranded 3 '-nucleotide C14. Also, PB1 residues $\mathrm{Arg}^{126}$ $(3.4 \AA), \operatorname{Ala}^{242}(2.8 \AA), \operatorname{Thr}^{243}(2.5 \AA), \operatorname{Arg}^{249}(2.9 \AA)$ and Gly $^{410}$ of Motif B loop (3.1 $\AA$ ) shows contacts with G16 while, $\operatorname{Asn}^{413}$ (2.9 and $2.8 \AA$ ) and $\mathrm{Glu}^{256}$ (2.8 $\mathrm{\AA}$ ) interacts with $\mathrm{C} 17$ of single stranded 3'-extremity of the activator strand. However, only two residues from PB2 $\mathrm{Arg}^{38}$ (3.2 and 
Fig. 2 Catalytic site and motifs of A/PR/8/34/H1N1 RNA dependent RNA polymerase predicted using MOE software suite
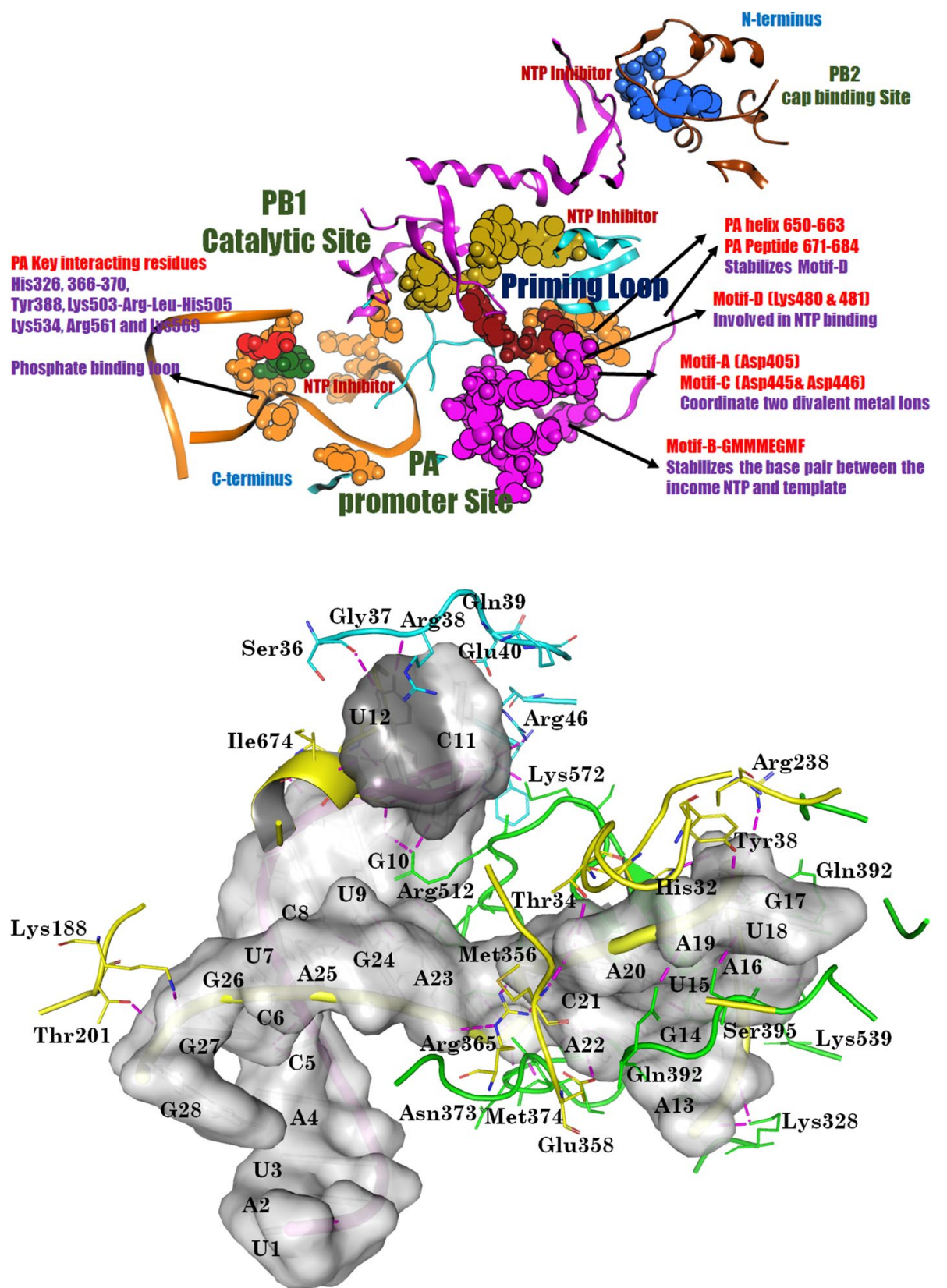

Fig. 3 Electrostatic interactions of the vRNA promoter with the homology model of IAV RdRp from A/PR/8/34/H1N1 strain. IAV RdRp domains PA, PB1 and $\mathrm{PB} 2$ are indicated in green, yellow and cyan colours and their hydrogen bonding contacts with vRNA promoter are represented in pink colour
$2.8 \AA$ ) and Lys $^{41}$ (3.3 $)$ are involved in binding to the U15 backbone phosphate group.

Mapping the oligonucleotide TTCC from $\$ 6$ polymerase shows that the capped primer binds inside a tunnel lined with predominantly basic amino acids $\mathrm{Lys}^{121}, \mathrm{Arg}^{126}$ and $\mathrm{Arg}^{249}$ from PB1 and $\mathrm{Lys}^{33}, \mathrm{Arg}^{38}$ and $\mathrm{Lys}^{41}$ from PB2 that leads to the active site of $\mathrm{A} / \mathrm{PR} / 8 / 34 / \mathrm{H} 1 \mathrm{~N} 1$ polymerase (Fig. 4). The residues Lys ${ }^{121}$ and Lys $^{33}$ from PB1 and PB2 lies away from the active site of the polymerase at the other end of the tunnel. In addition, $\mathrm{Arg}^{126}$ and $\mathrm{Arg}^{249}$ from PB1 and $\mathrm{Lys}^{41}$ from PB2 resides close to the polymerase active site. The PB1 residues Lys ${ }^{121}-\mathrm{Gln}^{127}$ and $\mathrm{Lys}^{33}$-Lys ${ }^{41}$ close to motif F3 of PB1 forms the platform for the primer nucleotides 3' DT2, 3' DC3 and DC4. This causes DC4 to interact with $\operatorname{Gln}^{124}$ while the residues from $\operatorname{Gln}^{137}-\mathrm{Thr}^{141}$ of PB1 forms the roof of the furanose ring of 3'-DT2 contacting with Pro ${ }^{138}$ at of $2.99 \AA$. In addition, the methyl group of pyrimidine interacts with one of the oxygen atoms of 3'-U12 of the activator strand. The observed primer nucleotide DT1 is placed far away from the active site close to $\mathrm{Arg}^{670}$ of PB1 peptide (671-684) and allows pyrimidine nitrogen to interact with oxygen atom of $3^{\prime}$-UT2. On the other side, $\mathrm{Arg}^{249}$ from the PB1-linker (196-257) which covers the external face of the PB1 fingers and palm domain form the terminal end for primer nucleotide DC4. Apart from the above, $\operatorname{Arg}^{126}$ stays close to the primer nucleotide DC3 without showing any 
Fig. 4 Surface representation of the $\Phi 6$ polio vRNA primer template in the homology model of IAV RdRp from A/PR/8/34/ H1N1 strain. PA, PB1 domains are represented in red, and green colours. Both GTP1 and GTP2 are indicated in orange and cyan colours. $\mathrm{Mg}^{2+}$ ions are represented in light green colour balls

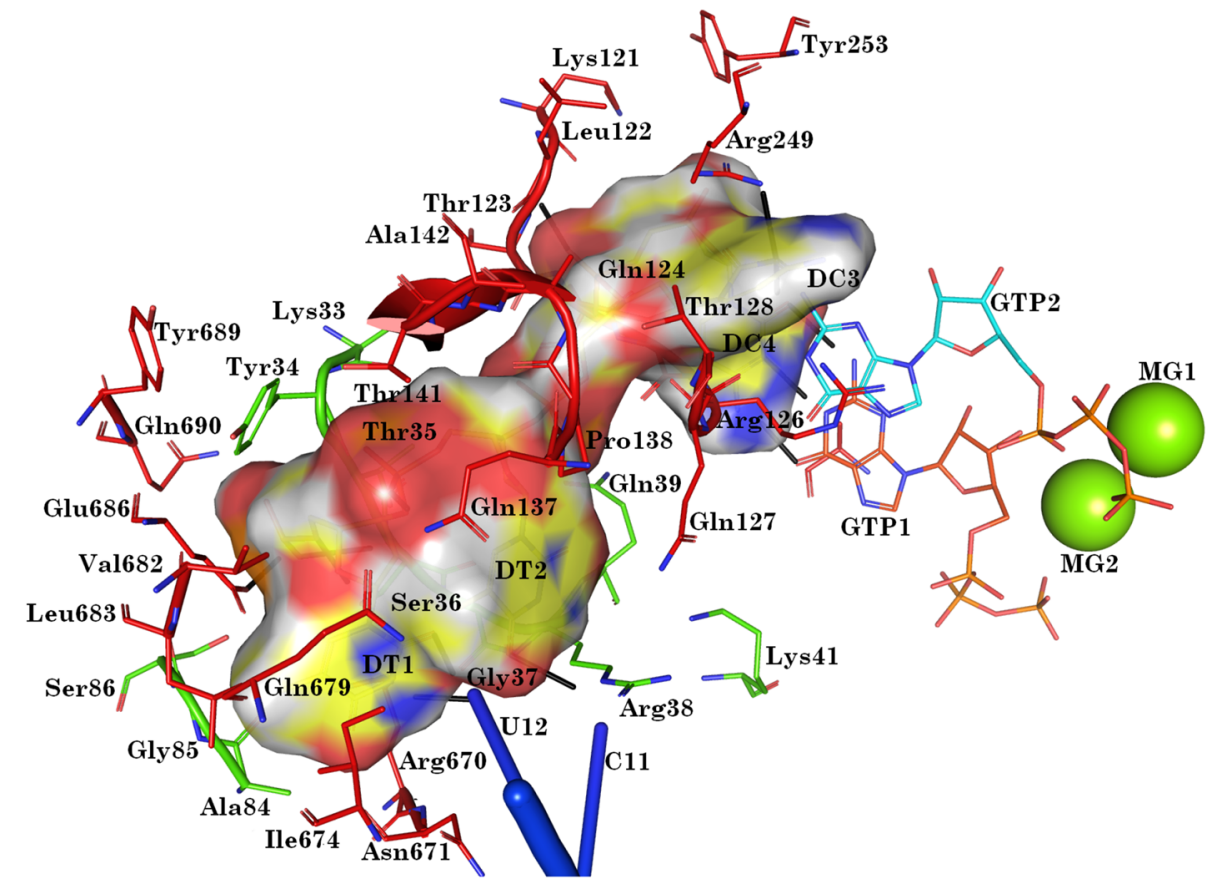

steric hindrances. The mapping studies also shows that the oligonucleotide TTCC lies exactly in parallel to the 3 '-terminal nucleotides 13 UCUGC17 of the influenzaB vRNA activator strand with a distance of $\sim 10.44 \AA$ between the backbone oxygens of DT2 and CT14. This predicts that the 3'-terminal nucleotides 13UCUGC17 of influenzaB vRNA activator may act as complementary base pairs for the capped RNA primer that transfer from the active site of polymerase endonuclease into the active site of the polymerase through the product exit channel (Fig. 5). Further superimposition of the poliovirus elongation complex (PDD: 1HI0) with $\mathrm{A} / \mathrm{PR} / 8 / 34 / \mathrm{H} 1 \mathrm{~N} 1$ polymerase places the $\mathrm{PB} 1$ $\beta$-hairpin tip (PB1 642-658) close to the 3'-sugar base of the initiating nucleotide GTP1 (Fig. 6). In addition, the terminal phosphate of GTP1 interacts with $\mathrm{His}^{649}$ and $\mathrm{Ala}^{648}$ at the tip of the priming loop. Additionally, the imidazole ring of guanosine shows H-Pi stacking with pro ${ }^{651}$ stabilized by the interaction between nitrogen and $\mathrm{Met}^{646}$. The terminal oxygen of the guanine nucleobase also contacts with DC4 of the capped primer. This shows clearly that the $\beta$-hairpin of PB1 might support internal initiation without obstructing the progress of the template strand.

Modeling with the polio template product elongation complex allows the 3'-extremity of the primer nucleotides DC 3 and DC4 to base pair with the 5'nt $11-13$ of the complementary RNA (cRNA) pulling the UCUCU of 5'-end of the cRNA towards the active site (Fig. 7). Furthermore, the vRNA showed a steric inherence at the PB2 N terminal domain with two stranded parallel $\beta$-ribbons ( $\beta 4-\beta 7$ and $\beta 5-\beta 6$ ) from residues Lys ${ }^{214}-\mathrm{Leu}^{218}$ and the outgoing template is blocked by helices $\alpha 8-\alpha 10$ of the PB2 lid

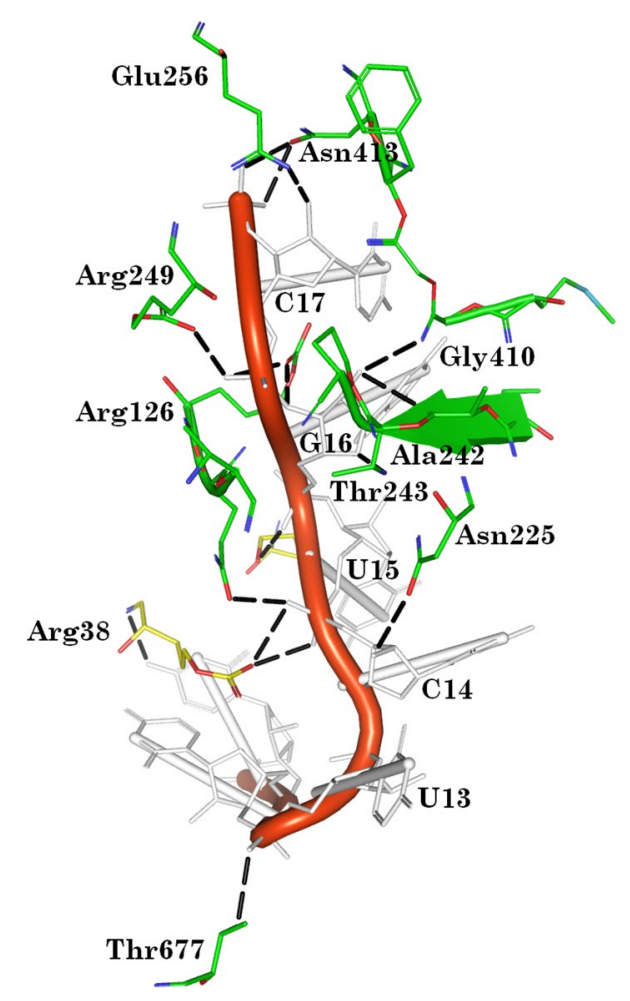

Fig. 5 Electrostatic interactions of 13UCUGCU18 bases of the activator strand of the vRNA after translocation into the homology model active site of IAV RdRp from A/PR/8/34/H1N1 strain

domain exhibiting showing seven hydrogen bonds with the terminal nucleotide $\mathrm{C} 1$. The residues of the helical lid, $\mathrm{Arg}^{175}$, Thr ${ }^{178}$ and $\mathrm{Gln}^{182}$ interacts with three hydrogen 
Fig. 6 Hydrogen bonding interactions of the incoming GTP with the homology model active site of IAV RdRp from $\mathrm{A} / \mathrm{PR} / 8 / 34 / \mathrm{H} 1 \mathrm{~N} 1$ strain

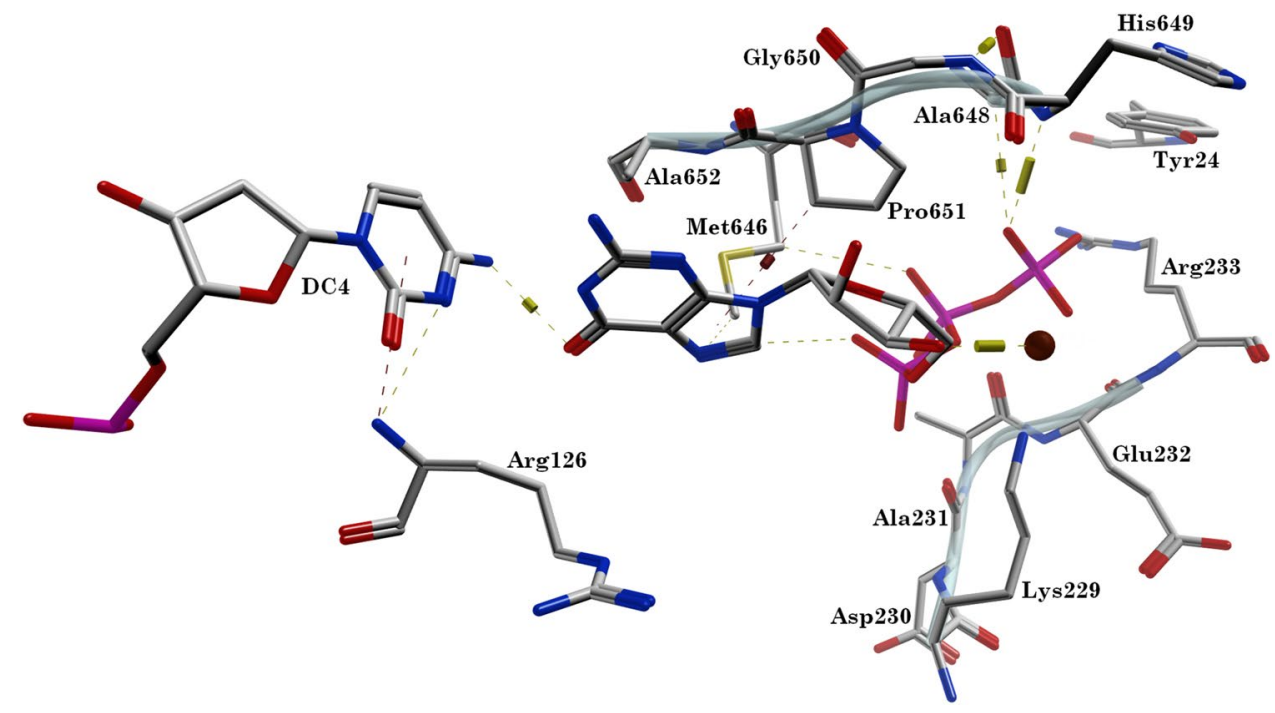

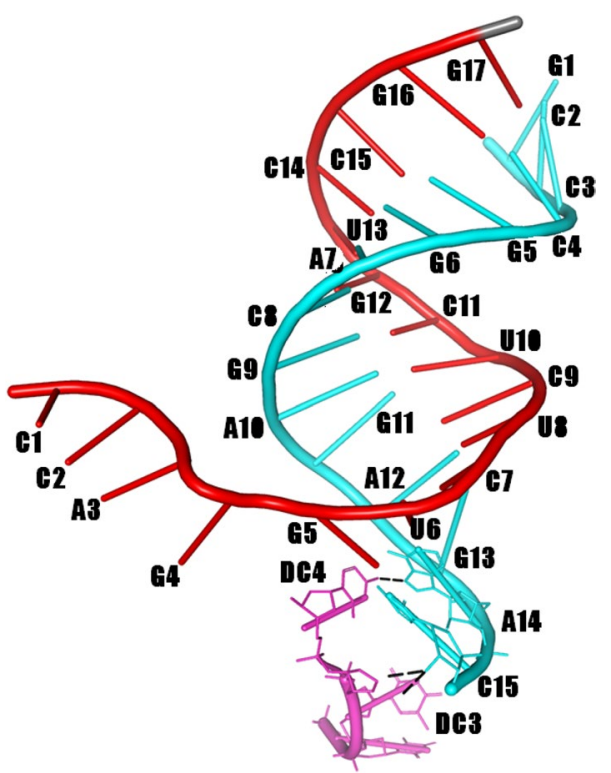

Fig. 7 Hydrogen bonding contacts between the 5'-elongation and $3^{\prime}$-primer templates from $\Phi 6$ vRNA polymerase mapped on to the homology model of IAV RdRp from A/PR/8/34/H1N1 strain

bonds at 3.5, 3.0 and 2.9 $\AA$ while, the residues of the PB2N2 subdomain Ser ${ }^{155}$ and $\mathrm{Glu}^{158}$ shows four other contacts with the backbone phosphate group at 2.4, 2.7, 3.4 and $2.8 \AA$ respectively. However, Lys ${ }^{157}$ of PB2-N2 subdomain contacts with sub-terminal nucleotide $\mathrm{A} 3$ at a distance of 3.1 $\mathrm{A}$. Furthermore, the residues $\mathrm{Tyr}^{205}, \mathrm{Glu}^{208}$, and $\mathrm{Arg}^{209}$ of the helical bundle ( $\alpha 9-\alpha 11$, residues $160-212)$ makes seven hydrogen bonds with A10 and G11, while Lys ${ }^{214}$ and $\mathrm{Thr}^{215}$ of $\beta 4$ shows contact with G9 of the outgoing template. On the other hand, $\operatorname{Arg}^{213}$ of $\beta 4$ shows contact with C14 of the cRNA at the $5^{\prime}$-extremity, while Asp ${ }^{146}$ of $\beta 5$ interacts with both C14 of cRNA and G6 of the outgoing template at the 5 -extremity of the elongation template. The residues of the linker region $\mathrm{Arg}^{216}{ }^{2} \mathrm{Leu}^{218}$ \& $\mathrm{Ala}^{221}-\mathrm{Gln}^{226}$ almost cover the major grove of the duplex RNA near the exit channel while the residues from Phe ${ }^{130}$-His ${ }^{151}$ pass between the duplex RNA from the exit channel towards the PB2-N2 subdomain of the helical bundle. The residues of the NLS domain cover the 5 '-end of the outgoing template showing three hydrogen bonding contacts with the 5 '-terminal nucleotide G17. Finally, two helices from PB1, Glu ${ }^{109}-\mathrm{Gln}^{127}$ and $\mathrm{Arg}^{249}-\mathrm{Glu}^{264}$ appear very close to the outgoing template near G4 and G5 without any contacts with cRNA, while the residues of the PA_C terminal helix Glu ${ }^{436}$ and $\mathrm{Val}^{432}$ stay very close to the 3'-terminal nucleotide of the vRNA at the exit channel. Energy minimization of the enzyme vRNA complex displaces the cRNA of the elongation template and penetrates between the priming loop without causing any steric inherences with the amino acid side chains. This helps the $\beta$-hairpin to stabilize the vRNA for internal initiation and viral replication by stabilizing the assembly of the initiation complex. In addition, RNA elongation causes structural changes of the two-stranded parallel $\beta$-ribbons ( $\beta 4-\beta 7$ and $\beta 5-\beta 6)$ to unfold which may prevent steric hindrances allowing vRNA to further pass through the exit channel (Fig. 8). Overall, our homology model not only satisfy previous experimental results but also clearly shows all the interactions between vRNA and IAV RdRp responsible for initiation and elongation phases of IAV replication. 


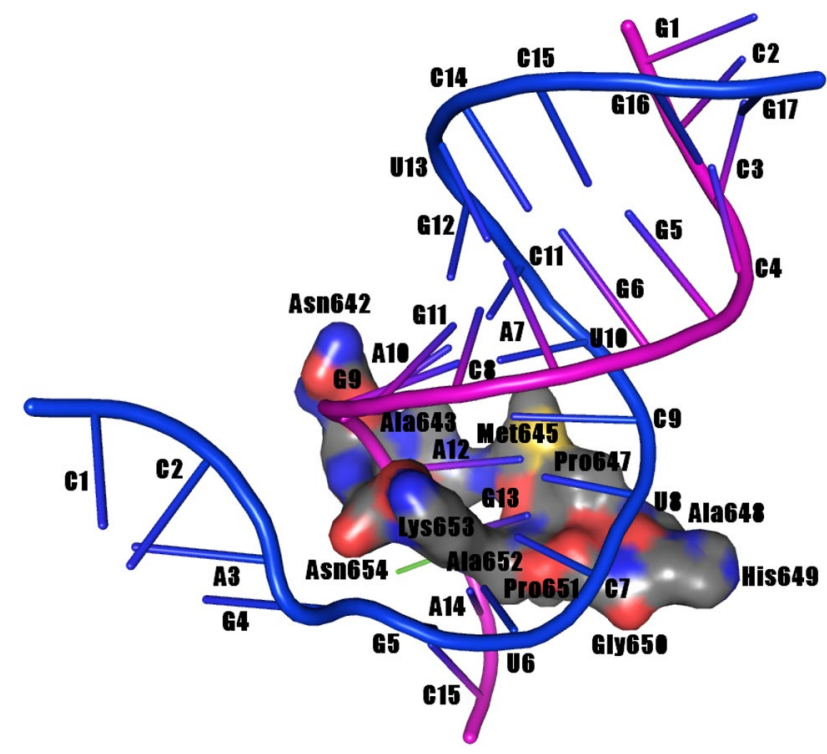

Fig. 8 Electrostatic interactions of elongation template of $\Phi 6$ vRNA polymerase with the priming loop of IAV RdRp homology model from $\mathrm{A} / \mathrm{PR} / 8 / 34 / \mathrm{H} 1 \mathrm{~N} 1$ strain

\section{Docking of known influenza polymerase drugs and AZT-TP with the homology model of $A / P R / 8 / 34 / H 1 N 1$ RdRp}

\section{Model I: In the presence of influenza-A vRNA}

Initial docking studies showed that favipiravir binds to the pocket located in the cleft between the helices $\alpha 3$ and $\alpha 12$ of the PB1 central domain and the 5'-hook of the template strand. The pyrazine base and carboxyamide orients opposite to each other towards the $\mathrm{N}$-and $\mathrm{C}$-terminal ends at approximate distances at 3.23 and $2.77 \AA$ distances. This orientation allows pyrazine nitrogen (N5) and carboxymide to interact with $\mathrm{Asp}^{41}$ and $\mathrm{Thr}^{385}$ of PB1 with $\Phi \mathrm{T}=145.4^{\circ}$ and $\Phi \mathrm{P}=-172.9^{\circ}$ (Fig. 9a). In contrast, $\mathrm{T}-705$ binds at the end of the tunnel II in the pocket between PB1 and PB2627 with $\Phi T=-151.7^{\circ}$ and $\Phi P=51.6^{\circ}$. The pyrazine carboxymide projects deeper into the cleft with fluorine pointing towards the PB2 627 linker and hydroxyl towards Arg ${ }^{287}$ of PB1 $\alpha 9$ interacting with met ${ }^{290}$ at a distance of $4.31 \AA$. In addition, the pyrazine ring also shows H-Pi interaction with PB1 Tyr ${ }^{497}$ of $\beta 19$. Furthermore, the 2 '-hydroxyl group of ribose along with its triphosphate contacts with $\mathrm{Arg}^{287}$ $(2.84 \AA), P^{501}(2.76 \AA)$ in the loop between $\beta 19$ and $\alpha 20$ of PB1, $\operatorname{Arg}^{142}(2.99 \AA)$ of PB2 N-terminal and Gly ${ }^{669}(2.87 \AA)$ of the PB2 627 linker. This shows that T-705 is surrounded by mostly positively charged and aromatic residues (Fig. 9b). Deflourination allows T-1105 to bind between the vRNA strands near the PA_C domain. This causes pyrazine and carboxymide (C6) to contact with Glu ${ }^{538}$ of PA_C and the 3'-hydroxyl group of the G2 5'-template strand with the distances of $\sim 2.68$ and $2.78 \AA$. The $1^{\prime}$-carbon of the ribose sugar also interacts with O6' ( $3.43 \AA$ ) and phosphate oxygen $(2.81 \AA)$ of $\mathrm{G} 25^{\prime}$-template strand with $\Phi \mathrm{T}=134.2^{\circ}$ and $\Phi \mathrm{P}=-52.3^{\circ}$. However, the terminal oxygen of the $\beta$ phosphate shows contacts with A4 nitrogen's N1 (2.71 $⿱$ ) and $\mathrm{N} 2(2.74 \AA)$ while the $\gamma$ phosphate interacts with G5 of the 5 '-template strand through C8 along with $\mathrm{Asp}^{697}$ (3.26 $\AA$ ) of the PA_C domain (Fig. 9c). Replacing the pyrazine hydroxyl group of T-1105 with oxo, allows the compound (T-1106) to bind in a similar orientation to T-705 in the second tunnel of the IAV polymerase. Instead, the carboxymide shows contacts with $\mathrm{Thr}^{487}(2.47 \AA)$ of PB1, $\mathrm{Asn}^{652}(2.89 \AA)$ through nitro and oxo while the pyrazine ring interacts with $A_{s n}{ }^{652}$ $(2.89 \AA)$ and $\operatorname{Asn}^{657}$ (2.97 $⿱$ ) of the PB2 627 linker domain keeping both domains intact. The $\alpha$ and $\gamma$ phosphates show contacts with Gly ${ }^{500}$ of PB1 (2.89 and $2.64 \AA$ ), Gly ${ }^{669}$ of PB2_C (3.28 $⿱$ A) and Lys ${ }^{140}(2.88$ and 3.0 $⿱$ ) of PB2_N with $\Phi \mathrm{T}=-178.6^{\circ}$ and $\Phi \mathrm{P}=1.5^{\circ}$ (Fig. 9d). Similarly, ribavirin triphosphate also binds at the same site similar to T-1106 but in an opposite orientation. The triazole and ribose sugar orients towards the $\mathrm{N}$-terminus of PB2 interacting with $\mathrm{Arg}^{142}$ $(2.84 \AA)$, Leu $^{668}(3.27 \AA)$ and Gly ${ }^{669}(3.13 \AA$ ) of the PB2_Nand $\mathrm{C}$-terminal domains through carboxamide oxo, $\mathrm{C} 2$ of pyrazine and the $C 1^{\prime}$ of ribose sugar with $\Phi T=81.4^{\circ}$ and $\Phi \mathrm{P}=156.4^{\circ}$. Furthermore, the $\alpha$ and $\beta$ phosphates towards the PB1 central domain interacts with the hydroxyl group of $\mathrm{Tyr}^{497}$ and $\mathrm{Pro}^{654}$ of the cyclic ring with distances of $\sim 2.73$, 2.78 and $3.10 \AA$ (Fig. 9e). In its dephosphorylated form, ribavirin occupies the binding site between $\mathrm{PB} 2 \_\mathrm{N}$ terminal and NLS domains with $\Phi T=77.9^{\circ}$ and $\Phi \mathrm{P}=150.7^{\circ}$. The $2^{\prime}$-hydroxyl group of ribose sugar orients towards the PB2 Nterminal showing contact with $\operatorname{Arg}^{136}$ while its $3^{\prime}$-hydroxyl group interacts with the back-bone carbon of $\mathrm{Ile}^{539}$ with the distances of 4.26 and $2.94 \AA$. In addition, the triazole carbons $2^{\prime}, 3^{\prime}$ and $4^{\prime}$ along with the $5^{\prime}$ oxygen interacts with $\mathrm{Glu}^{241}(3.06 \& 2.82 \AA)$ and $\mathrm{Glu}^{538}(3.18 \AA$ ) while the triazole-3-carboxamide orients towards the NLS domain interacting with $\mathrm{Ile}^{539}(3.72 \AA)$ and $\mathrm{Asn}^{540}(3.47 \AA)$ through their nitrogen atoms. The triazole also shows H-Pi interaction with the bone nitrogen atom of $\mathrm{Asn}^{540}$ of the NLA domain (Fig. 9f). In comparison, the prodrug viramidine binds at the surface of the template vRNA with ribose pointing towards the 5'-hook and triazole-3 carboxymide facing towards the PA_C domain. This allows the 6 '-hydroxy group to interact with phosphate oxygen and the 3 '-carbon of A4 while its $5^{\prime}$ and $1^{\prime}$ carbons contacts with Ala ${ }^{651}$ of the PA_C domain with distances of 2.71 and $3.07 \AA$. In addition, the triazole also shows H-Pi and hydrogen bonding with Lys ${ }^{574}$ of the PA_C domain with $\Phi T=178.9^{\circ}$ and $\Phi P=104.8^{\circ}$ (Fig. 9g). Similarly, AZT-TP also shows H-Pi stacking between the thymidine ring, $\mathrm{Arg}^{45}$, and $\mathrm{Arg}^{239}$ of motif F3 involved in the formation of the putative NTP tunnel. Also, thymidine and 


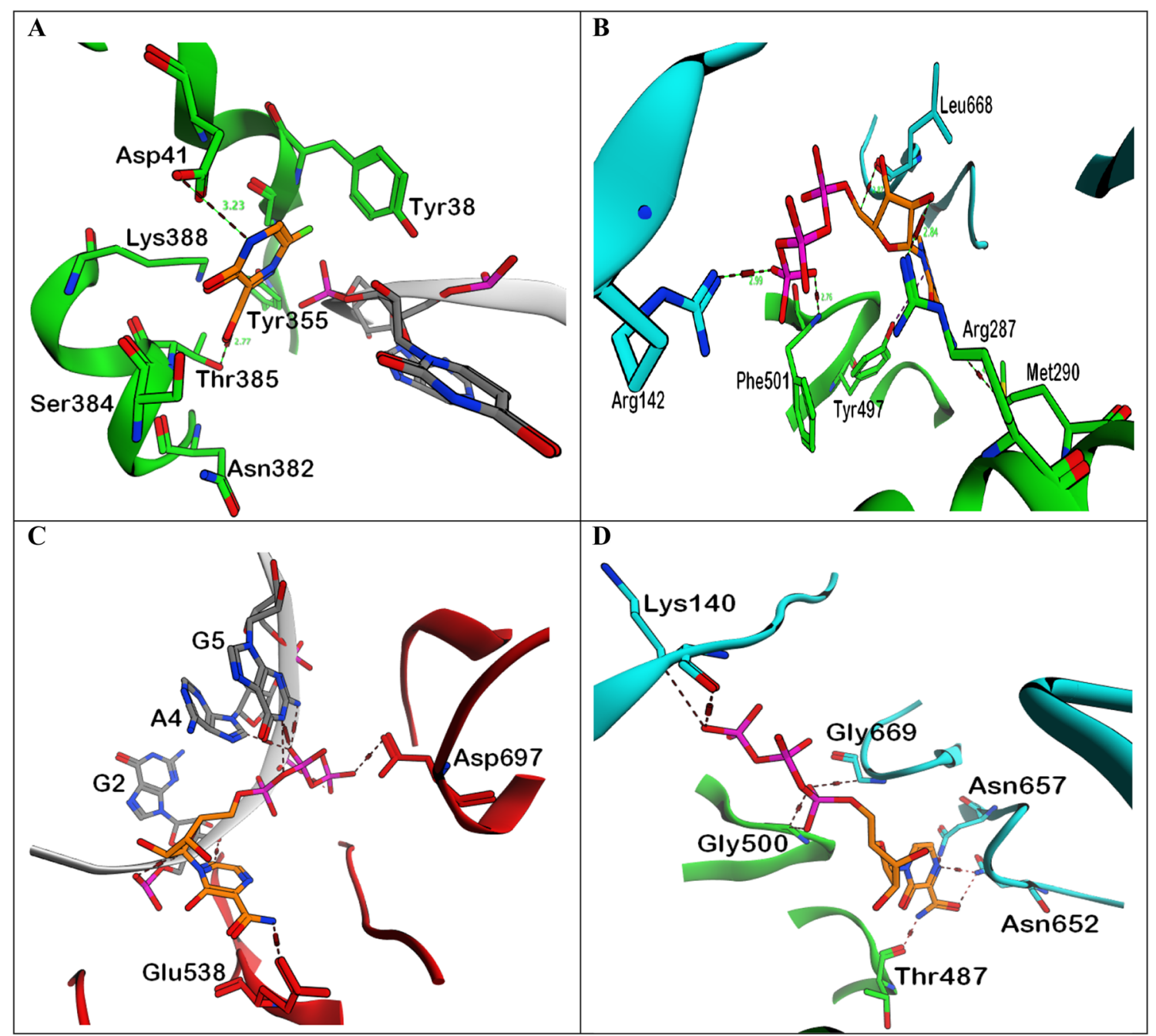

Fig. 9 Ligand protein interaction of Favipiravir (a), T-705 (b), T-1105 (c), T-1106 (d), Ribavirin Triphosphate (e), Ribavirin (f) Viramidine (g), AZT-TP (h) and 2FdGTP (i) with the homology model of IAV RdRp from A/PR/8/34/H1N1

its ribose sugar shows contacts with $\mathrm{Lys}^{235}, \mathrm{Ly}^{237}$ and $\mathrm{Arg}^{239}$ though the terminal oxo and backbone oxygen. Additionally, the terminal oxygen atoms of the $\beta$-phosphate interact with $\mathrm{Met}^{409}$ and Gly ${ }^{410}$ of the motif-B methionine-rich loop through the back-bone nitrogen and the side chain $\alpha$-carbon (Fig. 9h). This binding orientation clearly shows that AZTTP orients into the NTP tunnel with the triphosphate projecting towards the PB1 priming loop with $\Phi T=178.1^{\circ}$ and $\Phi \mathrm{P}=14.1^{\circ}$. In comparison, the purine group of $2 \mathrm{FdGTP}$ projects deeper into the cleft with the hydroxyl pointing towards the PB2 627 yielding a stronger hydrogen bond with PB2 $\mathrm{Lys}^{670}$. In addition, the imidazole ring shows contacts with PB2 $\mathrm{Thr}^{238}$ with two weaker hydrogen bonds at distances of 3.16 and $3.86 \AA$. Furthermore, the hydroxy groups of triphosphates show contacts with $\operatorname{Ser}^{594}$ (3.45 $\AA$ ) of PA, $\mathrm{Ala}^{503}$ (3.01 $\AA$ ) of PB1, and $\mathrm{Thr}^{238}$ (3.08 $⿱$ ) of PB2 near to the catalytic site of IAV RdRp (Fig. 9i). This shows that 2FdGTP occupies the end of tunnel II in the pocket between the PB1 and PB2 627 domains with $\Phi T=-82.7$ and negative $\Phi P=163.0$.

\section{Model II: In the presence of influenza-A vRNA with extended primer}

In the presence of the extended primer from $\Phi 6$ polymerase, favipiravir binds in the cleft between 3 '-end of the activator strand, $\eta 12$ and PB2 $\alpha 4$ showing contacts between G10 ribose sugar $(3.58$ and $2.16 \AA)$ and the pyrazine ring through oxo and nitrogen with $\Phi T^{\circ}=-1.3$ and $\Phi P=172.1^{\circ}$ (Fig. $\mathrm{S} 3 \mathrm{~A})$. Instead, T-705 binds to the same site as shown in model-I with $\Phi \mathrm{T}^{\circ}=-95.6$ and $\Phi \mathrm{P}=172.8^{\circ}$. The ligand interacts with Lys ${ }^{140}$ through pyrazine C6 with $2.89 \AA$ A. Additionally, $\beta$ and $\gamma$-phosphates along with the backbone oxygen of $\gamma$-phosphate shows contacts with $\mathrm{Ala}^{280}(2.87 \AA), \mathrm{Arg}^{287}$ (3.40 $\AA$ ) and $\operatorname{Arg}^{142}$ (2.68 and $2.89 \AA$ ) of both PB1 and PB2 domains respectively. This shows that $\mathrm{T}-705$ mainly interacts 


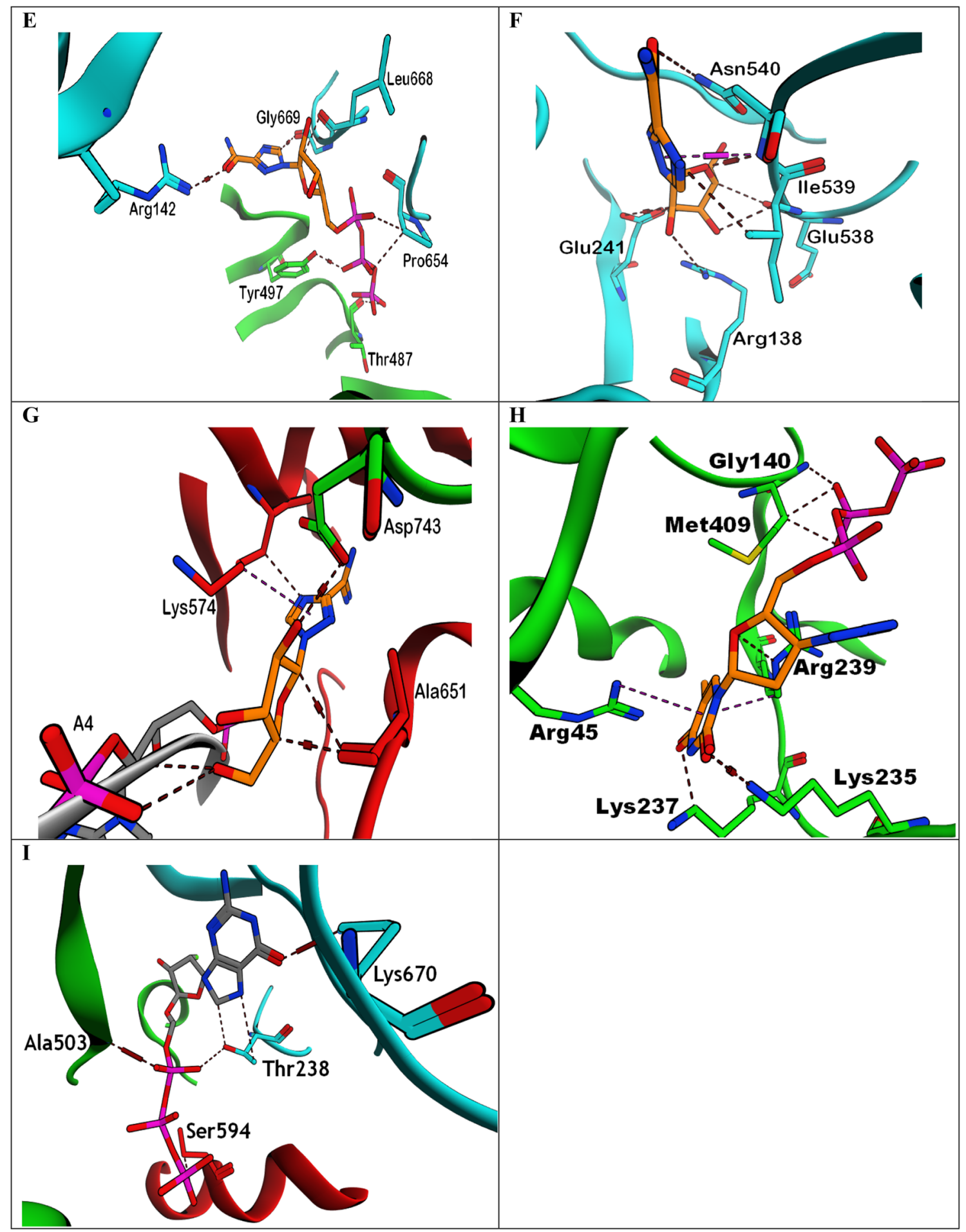

Fig. 9 (continued)

with positively charged residues at the end of tunnel-II below the PB2 627 linker domain (Fig. S3B). Defluorination allows the compound (T-1105) to bind with an orientation similar to the fluorinated compound T705. However, the pyrazine ring can approach more closely towards the PB2 NLS binding domain showing contact with $\mathrm{Glu}^{543}$ through the C6 carbon at a distance of $3.54 \AA$. Both $\beta$ and $\gamma$-phosphate oxygens along with the $\beta$-backbone oxygen contacts with Ile ${ }^{141}$ (2.71 and $2.95 \AA)$ and $\operatorname{Arg}^{142}(2.51 \& 3.0 \AA)$ of PB2 and $\operatorname{Arg}^{287}(2.67,2.76$ and $2.92 \AA)$ of PB1 with $\Phi T^{\circ}=-118.6$ 
and $\Phi P=-160.4^{\circ}$ (Fig. S3C). Further modification of the hydroxyl group with oxo, allows the compound (T-1106) to bind more towards the PB2 627 domain with $\Phi \mathrm{T}=-138.2$ and $\Phi P=170.2$. This orientation allows the pyrazine to contact with Gly ${ }^{500}$ and Gly ${ }^{669}$ of both PB1 and PB2 domains through oxo with distances of 3.22 and $3.0 \AA$. This in turn allows the attached ribose sugar to interact with $\mathrm{Val}^{139}$ (3.01 $\AA$ ) and Gly ${ }^{669}(2.57$ and $3.35 \AA)$ of PB2 and phe $e^{501}$ (2.64 and $2.73 \AA$ ) of PB1 through the 2'- and 3'-hydroxy groups including the $6^{\prime}$-carbon. This conformation allows the $\gamma$-phosphate to move freely in the binding pocket without showing any contacts with the viral polymerase. However, the $\alpha$ and $\beta$ phosphates shows contacts with $\operatorname{Ly}^{279}(2.64 \AA)$ and $\mathrm{Phe}^{501}$ (2.59 and $3.37 \AA$ ) of PB1 and $\operatorname{Arg}^{142}(2.82 \AA)$ of PB2_N with both terminal and backbone oxygens (Fig. S3D). Similarly, ribavirin triphosphate also binds at the same site interacting more or less with the same residues as for the other compounds shown above. The nitro and oxo groups of triazole interacts with $\mathrm{Tyr}^{499}$ of the PB1 central domain and Leu ${ }^{688}$ of PB2 627 at a distance of 2.55 and $2.90 \AA$ A. Both the 2 '- and 3'-hydroxyl groups of ribose sugar also contacts with Phe ${ }^{501}$ of PB1 and Arg ${ }^{142}$ of PB2_N with 2.61 and $3.13 \AA$ distances. In addition, the $\alpha$ and $\beta$ phosphates also interact with $\operatorname{Lys}^{140}(2.84 \AA), \operatorname{Arg}^{142}(2.80,2.73$ and $3.52 \AA$ ) of PB2 and $\operatorname{Arg}^{287}$ (2.74 and $3.07 \AA$ ) of PB1 with $\Phi T=-157.7^{\circ}$ and $\Phi P=162.4^{\circ}$ (Fig. S3E). In comparison, the dephosphorylated form of ribavirin also binds to the pocket between the 3 '-end of the activator strand, $\eta 12$ and PB2 $\alpha 4$ similar to favipiravir. In this case, the ribose sugar orients towards the activator strand and shows contacts with $\operatorname{Asn}^{671}(2.63 \AA)$ of PB1, G10 (2.94 ̊) and C11 (3.13 $\AA$ ) of the $3^{\prime}$-activator strand via $3^{\prime}$ and $4^{\prime}$ carbons along with the $4^{\prime}$ hydroxyl group. In addition, the triazole also interacts with $\operatorname{Pro}^{56}(3.16 \AA)$ and $\operatorname{Met}^{81}(4.01 \AA)$ through pyrazine and carboxamide nitrogens with $\Phi \mathrm{T}=-17.0^{\circ}$ and $\Phi \mathrm{P}=158.0^{\circ}$ (Fig. S3F). However, viramidine binds at the end of the exit channel between PA_C and PB2 627 domains with the ribose sugar facing towards the $\mathrm{PB} 2$ linker domain with $\Phi \mathrm{T}=-159.1^{\circ}$ and $\Phi \mathrm{P}=-132.4^{\circ}$. This orientation of ribose allows the $2^{\prime}$ and $3^{\prime}$ hydroxy groups along with the $2^{\prime}$ and $3^{\prime}$ carbons to interact with $\operatorname{Ser}^{601}(3.22 \AA)$ and $\mathrm{Asp}^{671}$ $(2.78 \AA)$ of both PA_C and PB2 627 domains. In addition, the 3-carboximidamide nitrogen shows contact with $\mathrm{Ser}^{594}$ of PA_C domain $2.82 \AA$ away (Fig. S3G). Similarly, AZT-TP penetrates deeply below the PB2 627 linker domain showing interaction with $\mathrm{Arg}^{664}$ through the oxo group of thymine. The $\alpha$ and $\gamma$-triphosphates also interact with $\mathrm{Phe}^{501}$, Lys ${ }^{140}$ and $\mathrm{Thr}^{238}$ of PB2_N through the terminal oxygen. Both the thymidine base and ribose sugar also show interactions with PB2 Asn ${ }^{137}$ through their backbone carbon and oxygen atoms. In addition, the azido group also makes contacts with $\mathrm{Val}^{502}$ of PB1 central domain (Fig. S3H). This shows that AZT-TP mostly occupies tunnel2 near to the PB2 linker domain. In comparison, the purine group of $2 \mathrm{FdGTP}$ projects deeper into the cleft below the PB2 627 linker domain with the hydroxyl pointing opposite to PB2 627 showing stronger hydrogen bond with Asp ${ }^{538}$ of PB2 $3.02 \AA$ away. In addition, the furanose ring shows contacts with the PB1 Phe ${ }^{501}$ and $\mathrm{Val}^{502}$ with two stronger hydrogen bonds at distances of 2.91 and $2.86 \AA$ respectively. Furthermore, the terminal hydroxy group of triphosphate interacts with Lys $^{670}(3.40 \AA)$ of the PB2 linker (Fig. S3I). This shows that, 2FdGTP occupies the end of the tunnel II in the pocket between the PB1 and PB2 627 domains similar to model-I with $\Phi \mathrm{T}=168.7^{\circ}$ and $\Phi \mathrm{P}=-78.7 .1^{\circ}$.

\section{Model III: In the presence of influenza-B vRNA}

In the presence of influenzaB vRNA, favipiravir binds deeper into the cleft between PA_C, PB1 central and PB2 627 domains contacting with $\mathrm{Asn}^{657}$ and $\mathrm{Asn}^{659}$ through oxo and pyrazine nitrogen with $\Phi \mathrm{T}=-33.4^{\circ}$ and $\Phi \mathrm{P}=169.3^{\circ}$ (Fig. S4A). Surprisingly, T-705 binds between the $3^{\prime}$-end of the activator strand, $\eta 12$ and PB2 $\alpha 4$ which is completely different in comparison to model-I and model-II with $\Phi \mathrm{T}=-177.1^{\circ}$ and $\Phi \mathrm{P}=-169.1^{\circ}$. In this orientation, the carboxymide nitrogen shows contacts with $3^{\prime} \mathrm{C} 14$ (2.48 and $2.63 \AA$ ) and U15 (3.24 $\AA$ ) through $2^{\prime}$ hydroxyl and the backbone phosphate oxygen, while the oxo group shows contact with $\operatorname{Arg}^{135}(2.82 \AA)$ and $\operatorname{Asn}^{136}(2.88 \AA)$ of the PB1 central domain above the 3 '-end of the vRNA through $\alpha$ carbon and back bone nitrogen bonding. In addition, the pyrazine nitrogen (N4) also interacts with $\mathrm{Arg}^{135}$ of PB1 through the $\alpha$ carbon with a distance of $2.91 \AA$. On the other hand, the ribose sugar and the attached $\gamma$-phosphate shows contacts with $3^{\prime}$ U12 (2.89 and $\left.3.0 \AA\right)$ and U13 (3.25 $\AA$ ), $\operatorname{Gln}^{137}(2.65 \& 2.88 \AA), \operatorname{Pro}^{138}(3.22 \AA)$ and $\mathrm{Ala}^{140}(2.95 \AA)$ of PB1 through 2' and $3^{\prime}$-hydroxy groups along with $3^{\prime}$ and $6^{\prime}$-carbons (Fig. S4B). Defluorination allows the compound (T-1105) to bind between the two vRNA strands near to the PA_C domains with $\Phi T=-153.9^{\circ}$ and $\Phi P=-164.3^{\circ}$. In this binding orientation, the pyrazine ring forms $\mathrm{H}-\mathrm{Pi}$ interaction with $\mathrm{Tyr}^{30}$ of PB1 through the $\alpha$-carbon with additional contact with Ile ${ }^{573}$ (3.78 $⿱$ A) of PA_C domain through N4 nitrogen. Simultaneously, the ribose sugar interacts with $\operatorname{Asn}^{513}(3.13,2.77 \& 3.12 \AA)$ of the PA_C domain using 1 '- and 4'-carbons including the 2'-hydroxy group. Apart from these contacts, the terminal oxygens of triphosphates also show contacts with $\mathrm{His}^{32}(2.69 \AA)$ of the PB1 second loop (24-38) which crosses from thumb to fingers through the backbone oxygen. Additionally, the triphosphate oxygens also contact with $\mathrm{C} 14(2.84,3.56$ and $2.75 \AA)$ and U15 (3.42 $\AA$ ) of the 3 'vRNA activator stand through their back-bone oxygen and side chain oxo including the carbon of nucleobase $\mathrm{C} 14$ (Fig. S4C). Interestingly, the compound with an oxo group, T-1106 binds to the same site as shown 
in model-I and model-II with $\Phi \mathrm{T}=90^{\circ}$ and $\Phi \mathrm{P}=-177.9^{\circ}$. However, in this conformational change, the 3-oxo-3,4-dihydropyrazine-2-carboxamide does not show any contact with the IAV RdRP while the ribose sugar interacts with Lys ${ }^{670}$ $(3.03 \AA)$ and $\mathrm{Gly}^{669}(2.83 \AA$ ) of PB2 nearer to the NLS domain (685-760) via the 2 '-carbon and hydroxy groups. The $\alpha$ and $\gamma$ phosphates which orients towards the entry channel shows contacts with $\mathrm{Asn}^{16}(2.78 \AA), \mathrm{Ala}^{503}(2.74 \AA$ ), $\operatorname{Asp}^{538}$ (3.31 $\AA$ ) of PB1 and $\operatorname{Ser}^{594}$ (3.31 $\AA$ ) of PB2 of which Asp $^{538}$ and Ser ${ }^{594}$ of PB1 reside in the central region of the typical right-handed RdRp fold (Fig. S4D). Similarly, ribavirin triphosphate almost binds at the same site similar to $\mathrm{T}-1106$ interacting with $\Phi \mathrm{T}=-131.0^{\circ}$ and $\Phi \mathrm{P}=-173.6^{\circ}$. The triazole that orients towards the entry channel interacts with Gln ${ }^{591}$ of PA through the carboxymide nitrogen while the ribose sugar contacts with $\operatorname{Gln}^{669}\left(3.47 \AA\right.$ ) and Lys ${ }^{670}$ (3.34 ̊) of PB2 627 through 2'- and 6'-carbons. Furthermore, the $\beta$ and $\gamma$ phosphates also interacts with the residues of the central region, $\mathrm{Val}^{502}(2.88 \AA)$ and $\mathrm{Ala}^{503}(1.88 \AA)$ with their terminal and backbone oxygens (Fig. S4E). In comparison, the dephosphorylated form of ribavirin binds at the surface of the 5'-hook without showing any contacts with the template strand of the vRNA. The ribose sugar shows contacts with $\operatorname{Ser}^{648}(3.27 \AA), \mathrm{Leu}^{649}(2.59 \AA)$ and $\mathrm{Ala}^{651}$ (3.36, 3.25 and $2.96 \AA$ ) of PA_C through $1^{\prime}, 3^{\prime}$ and $6^{\prime}$-carbons including the $2^{\prime}$-hydroxyl group. In addition, triazole interacts with $\mathrm{Asn}^{466}(2.84 \AA), \mathrm{Ly}^{574}(3.03 \AA)$ and $\mathrm{Leu}^{649}$ $(4.07 \AA)$ of the PA-C domain through triazole and carboxymide nitrogen's (Fig. S4F). Furthermore, the ribavirin analog viramidine also binds similarly to the same binding site at the surface of the template vRNA with eleven hydrogen bonds. The 5-hydroxy methy shows $\mathrm{H}-\mathrm{Pi}$ and hydrogen bonding with G5 and A4 of the 5'-hook of template vRNA while the carbon and oxygen atoms of tetrahydrofuran show H-Pi and hydrogen bonds with $\mathrm{Ala}^{651}$ and $\mathrm{Arg}^{279}$ of PA_C domain. In addition, the triazole also shows H-Pi and hydrogen bond with Lys ${ }^{574}$ and Lys ${ }^{281}$ of PA_C domain and backbone oxygens of A4 of template vRNA 5'-hook (Fig. $\mathrm{S} 4 \mathrm{G})$. Overall, these binding orientations clearly indicate that favipiravir and its derivatives adopt a trans conformation

Table 2 Calculated torsional angles ( $\Phi \mathrm{T}$ and $\Phi \mathrm{P}$ ) for the known inhibitors of InfluenzaA RdRp in the presence of FluA vRNA

\begin{tabular}{lrr}
\hline Compound & \multicolumn{1}{c}{$\begin{array}{l}\text { DT } \\
\text { Deg }\end{array}$} & \multicolumn{1}{c}{ Deg } \\
\hline Favipiravir & 145.4 & -172.9 \\
T-705 & -151.7 & 51.6 \\
T-1105 & 134.2 & -52.3 \\
T-1106 & -178.6 & 1.5 \\
Ribavirin-TPT & 81.4 & 156.4 \\
Ribavirin & 77.9 & 150.7 \\
\hline
\end{tabular}

in all the four models. However, both phosphorylated and dephosphorylated forms of ribavirin adopt a cis conformation with a small deviation in both the dihedral angles in the presence of influenza-A vRNA (Table 2). However, both favipiravir and ribavirin shows a large deviation of $\Phi T$ in the presence of influenza-A vRNA with extended primer (Table 3) while only favipiravir adopts a large deviation of $\Phi T$ in the presence of influenza-B vRNA (Table 4) compared to other known compounds studied. The presence of thymidine allows, AZT-TP to interact with the backbone and side chain of $\mathrm{Ala}^{140}$ of the PB1 N-terminal region through 2 '-oxygen at a distance of 2.00 and $3.29 \AA$ respectively. In addition, the azido group also shows contacts with PB1 $\operatorname{Gln}^{127}(2.80 \AA), \operatorname{Thr}^{128}(3.33 \AA)$ and $\operatorname{Pro}^{138}(2.72 \AA)$ with three strong hydrogen bonds. However, the triphosphate projects towards the catalytic site contacting with PB1 Gly 125 and $\mathrm{C} 17$ backbone carbon and oxygen atoms at a distance of 2.70, 3.02 and $3.56 \AA$ respectively (Fig. S4H). This shows that AZT-TP mostly occupies away from the catalytic site adjacent to the 3 '-activator strand. In comparison, the purine group of 2FdGTP projects deeper into the cleft below the PB2 627 linker domain with amino nitrogen interacting with Phe ${ }^{501}$ of PB1 central domain (2.76 $⿱$ ). In addition, pyrazine nitrogen also contacts with $\mathrm{Val}^{503}(3.49 \AA$ ) of PB 1 central domain with a weaker hydrogen bond. However, furanose ring shows no contacts with either the PB1 or PB2 domains while the terminal and second hydroxy groups

Table 3 Calculated torsional angles ( $\Phi \mathrm{T}$ and $\Phi \mathrm{P}$ ) for the known inhibitors of InfluenzaA RdRp in the presence of FluA vRNA with extended primer

\begin{tabular}{lrr}
\hline Compound & \multicolumn{1}{c}{$\Phi \mathrm{T}$} & \multicolumn{1}{c}{$\Phi$ P } \\
& \multicolumn{1}{c}{ Deg } \\
\hline Favipiravir & -1.3 & 172.1 \\
T-705 & -95.6 & 172.8 \\
T-1105 & -118.6 & -160.4 \\
T-1106 & -138.2 & 170.2 \\
Ribavirin-TPT & -157.7 & 162.4 \\
Ribavirin & -17.0 & 158.0 \\
\hline
\end{tabular}

Table 4 Calculated torsional angles ( $\Phi T$ and $\Phi P$ ) for the known inhibitors of InfluenzaA RdRp in the presence of in the presence of FluB vRNA

\begin{tabular}{lrr}
\hline Compound & \multicolumn{1}{c}{$\Phi \mathrm{T}$} & \multicolumn{1}{c}{$\Phi \mathrm{P}$} \\
& \multicolumn{1}{c}{ Deg } & \multicolumn{1}{c}{ Deg } \\
\hline Favipiravir & -33.4 & 169.3 \\
T-705 & -177.1 & -169.1 \\
T-1105 & -153.9 & 164.3 \\
T-1106 & 90.0 & -177.9 \\
Ribavirin-TPT & -131.0 & 174.3 \\
Ribavirin & 125.9 & -173.6 \\
\hline
\end{tabular}


of triphosphates interacts with $\mathrm{Phe}^{501}(2.64 \AA)$ and $\mathrm{Val}^{503}$ ( $3.40 \AA$ ) of the PB1 central domain and $\operatorname{Arg}^{142}$ of PB2 N-terminal domain (Fig. S4I). This shows that, 2FdGTP occupies the end of the tunnel II in the pocket between the PB1 and PB2 627 domains similar to model-I and II with dihedral angles of $\Phi \mathrm{T}=56.5^{\circ}$ and $\Phi \mathrm{P}=-66.3^{\circ}$.

\section{Model IV: In the presence of influenza-A vRNA with extended primer and 3'-elongation template}

In the presence of the extended primer and the elongation strand, favipiravir binds close to catalytic site forming a Pistack with $\mathrm{Arg}^{126}$ of Motif B loop (3.58 and 2.16 $⿱$ A) with $\Phi T^{\circ}=-49.8$ and $\Phi P=173.8^{\circ}$ (Fig. S5A). Similarly, T-705, T1106, ribavirin and ribavirin-TP also occupy the same site with ligand protein interactions at different distances. T705 interacts with $\mathrm{Asn}^{413}$ through pyrazine nitrogen while the hydroxyl groups of the nucleobase show Pi-stacking with $\mathrm{C} 15$ of the 3 '-elongation strand. The sugar ring also shows contacts with $\operatorname{Gln}^{127}$ (3.41 $\AA$ ) $\operatorname{Pro}^{244}(2.87 \AA)$ and $\operatorname{Arg}^{249}$ $(2.96 \AA)$ of the PB1 central domain. Additionally, $\alpha$ and $\gamma$-phosphates shows contacts with $\operatorname{Arg}^{126}(2.52,2.93$ and $3.26 \AA$ ) and $\operatorname{Arg}^{249}$ (3.18 $\mathrm{A}$ ) of PB1 central domain respectively. (Fig. S5B). Defluorination allows T-1105 to bind to the PB1 catalytic site interacting with $\mathrm{Phe}^{495}$ (3.7), Gly ${ }^{234}$ $(3.33 \& 3.76 \AA), \operatorname{Lys}^{235}(2.87 \AA)$ and $\mathrm{Ala}^{648}(3.18 \AA)$ of the PB1 priming loop. In this conformation, the nucleobase orients towards the NTP tunnel while the triphosphate approach close to the priming loop placing the ribose sugar close to Lys ${ }^{664}(2.81$ and $3.03 \AA$ ) of the PA_C domain with $\Phi \mathrm{T}^{\circ}=141.5^{\circ}$ and $\Phi \mathrm{P}=-95.8^{\circ}$ (Fig. S5C). Replacing the hydroxyl group with oxo, allows T-1106 to bind more towards the PB1 central domain with $\Phi \mathrm{T}=-143.0^{\circ}$ and $\Phi P=-21^{\circ}$. This orientation makes the pyrazine to contact with $\mathrm{C} 15$ of the 3 '-elongation strand through oxo at a distance of $2.8 \AA$. This in turn allows the $\alpha$ and $\gamma$ phosphates to interact with $\operatorname{Pro}^{244}$ (3.27 $\AA$ ), $\operatorname{Arg}^{249}$ (3.11 $\mathrm{A}$ ) and $\operatorname{Tyr}^{253}$ $(3.10 \AA)$ of the PB1 central domain through terminal and backbone oxygens (Fig. S5D). Similarly, ribavirin triphosphate also binds to the same site interacting more or less with the same residues as for the other compounds shown above. The nitro and oxo groups of triazole interacts with Lys $^{121}$ of the PB1 central domain and C15 of 3'-elongation strand with 2.56 and $2.98 \AA$ distances. In addition, both 2 '-hydroxyl group and back bone oxygen atom of ribose sugar contacts with $\mathrm{C} 15$ of $3^{\prime}$-elongation strand and $\mathrm{Arg}^{249}$ with $3.26 \AA$ distances. This orientation in turn allows the tri phosphates to interact with $\operatorname{Pro}^{244}(2.70 \AA)$ ), $\operatorname{Arg}^{249}(3.50 \AA)$, $\mathrm{Phe}^{412}\left(3.78 \AA\right.$ ) and $\mathrm{Asn}^{413}(3.52 \AA)$ of PB1 central domain with $\Phi T=-166.3^{\circ}$ and $\Phi P=-52.4^{\circ}$ (Fig. S5E). In comparison, the dephosphorylated form of ribavirin also binds to the same pocket nearer to the 3 '-end of the elongation strand, similar to its triphosphate form. In this case, the ribose sugar orients close to the motif-B loop and shows contacts with $\mathrm{Leu}^{122}\left(2.63 \AA\right.$ ) and $\mathrm{Arg}^{249}$ of the PB1 central domain and C15 of $3^{\prime}$-elongation strand $(2.94 \AA)$ through $2^{\prime}$ and backbone oxygens. In addition, the triazole also interacts with $\operatorname{Arg}^{249}(3.16 \AA)$ through Pi-stacking with $\Phi \mathrm{T}=-117.1^{\circ}$ and $\Phi \mathrm{P}=131.2^{\circ}$ (Fig. S4F). Moreover, viramidine also binds at the same site as ribavirin interacting with the terminal nitrogen of the $\mathrm{C} 15$ elongation strand and $\mathrm{Gln}^{127}$ of $\mathrm{PB} 1$ motif-B loop through imidazole and the ribose sugar with $\Phi \mathrm{T}=-174.1^{\circ}$ and $\Phi \mathrm{P}=-60.8^{\circ}$ (Fig. S5G). However, AZT-TP binds to the PB1 catalytic site nearer to the priming loop interacting mainly with $\mathrm{Lys}^{481}(2.72,2.75,2.56$, $3.18 \& 3.63 \AA$ ) and $\operatorname{Ser}^{494}(2.88 \AA)$ through stronger hydrogen bonds (Fig. S5H). In addition, the thymidine oxo also contacts with the $\mathrm{Asp}^{305}$ carbon through a weaker hydrogen bond. In comparison, the purine group of 2FdGTP orients towards the 3'-end of the positive elongation strand showing weaker hydrogen bonding with Phe $\mathrm{P}^{412}$ of PB1 motif-B at a distance of $3.44 \AA$. In addition, the terminal hydroxy group of the triphosphate contacts with the terminal nitrogen atom of the $\mathrm{C} 15$ (3.08 $⿱$ ) elongation strand (Fig. S5I). This shows that, 2FdGTP also occupies a site nearer to the PB1 catalytic site above the elongation strand at the $3^{\prime}$-end with $\Phi \mathrm{T}=140.6^{\circ}$ and $\Phi \mathrm{P}=20.9^{\circ}$.

\section{Activity of AZT-TP and 2FdGTP on InfluenzaA Virus transcription}

Experimental studies using a purified influenzaA virus A/ $\mathrm{PR} / 8 / 34 / \mathrm{HIN} 1$ in an NTP incorporation assay with an RdRp enzyme showed that 2FdGTP, a known inhibitor of FluA polymerase exhibited a $25 \%\left(\mathrm{IC}_{25}\right)$ and $50 \%\left(\mathrm{IC}_{50}\right)$ effective concentrations at 0.3 and $1.2 \mu \mathrm{M}$ respectively. Similarly, AZT-TP (Fig. 10a, b) also inhibited the viral polymerase with an $\mathrm{IC}_{25}$ and $\mathrm{IC}_{50}$ at 0.2 and $1.2 \mu \mathrm{M}$ respectively.

\section{Discussion}

Here we report for the first time the binding modes of the known influenza polymerase drugs, Favipiravir, T-705TP, T-1105TP, T-1106TP, ribavirin, ribavirinTP, viramidine and 2FdGTP. In addition, we found that AZT-TP was also predicted to bind to our homologous model and this was confirmed in vitro polymerase transcription assays. We showed that AZT-TP binds to the homology model of the A/PR/8/34/ H1N1 influenza virus RdRp in four models 1 . In the presence of influenzaA $v$ RNA; II. In the presence of influenza-A vRNA with extended primer and III. In the presence of influenzaB vRNA and IV. In the presence of the RNA promoter including the initiation primer and the 3'-elongation template from polio virus. The docking results from all the four 

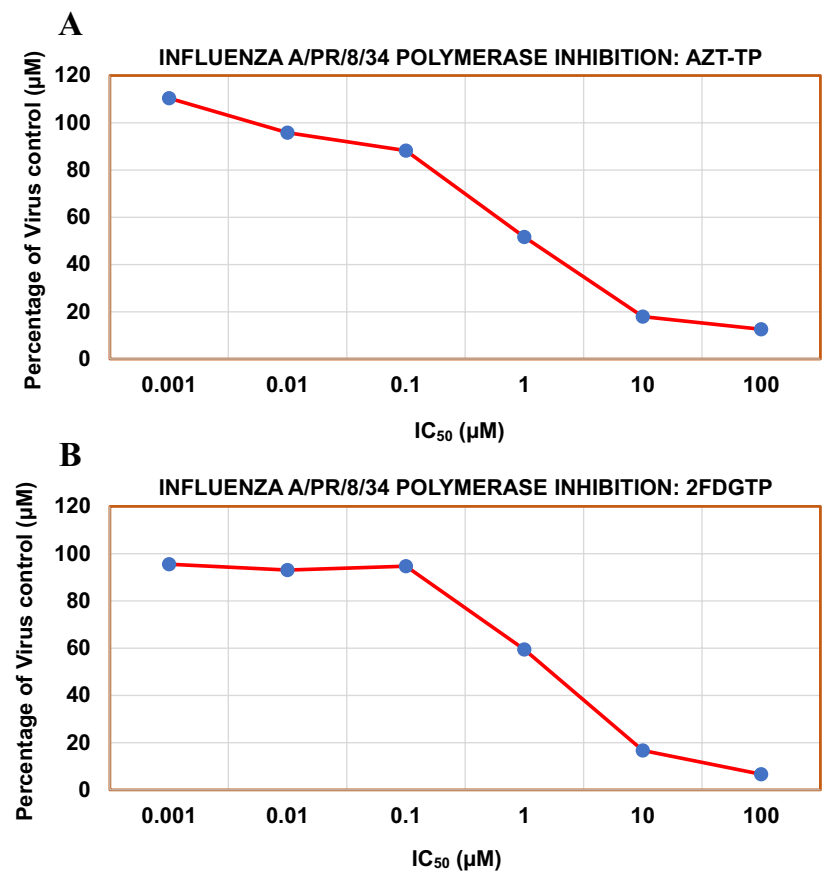

Fig. 10 Inhibition concentration $\left(\mathrm{IC}_{50}\right)$ of the IAV RdRp inhibition from $\mathrm{A} / \mathrm{PR} / 8 / 34 / \mathrm{H} 1 \mathrm{~N} 1$ strain with the compounds (a), 3'-azido-3'deoxy-thymidine-5'-triphosphate and 2'-deoxy-2'-fluoroguanosine triphosphate (b) using TCA precipitation assay. Percentage of virus inhibition is shown in Y-axis and Inhibitor concentration is shown in $\mathrm{y}$-axis and $\mathrm{IC}_{50}$ values are shown in $\mathrm{X}$-axis

models I, II, III and IV showed that 2'-deoxy-2'fluoroguanosnine 5 '-triphosphate binds at the end of the tunnel 2 with the highest binding affinity of $-16.3 \mathrm{Kcal} / \mathrm{mol}$ in model-I and inhibited the purified polymerase transcription with an $\mathrm{IC}_{50}$ of $1.12 \mu \mathrm{M}$. The inhibition mechanism of the influenza polymerase revealed that the compound 2'-fluorodGTP competitively inhibits incorporation of GMP into anti-genomic RNA. However, 2 '-fluorodGTP known not to be incorporated into the growing RNA strand but competitively inhibits incoming GTP to inhibit chain elongation [12]. Thus, our binding mode using docking in correlation with previous kinetic studies suggested that 2 '-fluorodGTP may be incorporated and may cause chain termination of further transcription. Similarly, T-705 has been shown to be a chain-terminator and competitive inhibitor inhibits capsnatching and transcription initiation when p32-labelled 5'-cap1 RNA was added to a virus derived RNA complex [47]. The higher observed polymerase inhibition of T-1106 with respect to T-705 correlates well with the higher binding energy of T-1106 $(-23.8 \mathrm{Kcal} / \mathrm{mol})$ over T-705 $(-21.08 \mathrm{Kcal} / \mathrm{mol})$ in model-I [48]. Furthermore, the lower predicted binding energy of ribavirin with $-19.3 \mathrm{Kcal} / \mathrm{mol}$ compared with T-705 $(-21.08 \mathrm{Kcal} / \mathrm{mol})$ using our model and docking studies correlates with data from previous polymerase inhibition assays showing a greater potency of T-705 than ribavirin $[49,50]$. This clearly reveals that favipiravir binds at the 3 '-end of the activator vRNA while its triphosphate derivatives T-705, T-1105 and T-1106, along with Ribavirin triphosphate binds in a cleft between PB2_N and NLS domain nearer the exit channel of the IAV RdRp. However, ribavirin binds to the PB2 627 linker domain while viramidine occupies the cleft near to the 5 '-hook of the vRNA template strand. Even after the translocation of the $3^{\prime}$-end of the activator strand into the active site, as shown in model-III, T-1105 occupies the 3'-end of the vRNA activator strand. In addition, T-1106 and ribavirin triphosphate but not favipiravir exhibits the same binding site nearer to the exit channel as shown in model-I. However, both ribavirin and its prodrug viramidine bind at the 5 -hook of the vRNA template strand. Since ribavirin inhibits viral replication through potential additional mechanisms including immunosuppression and lethal virus mutagenesis, the mode of action is rather complex [51, 52]. It was demonstrated that mutation of V43I in PB1 close to the putative NTP entrance channel affects the activity of ribavirin [52]. Our docking results also show that ribavirin has the ability to bind to the NTP tunnel close to $\mathrm{Thr}^{42}$ with a binding energy of $-10 \mathrm{kcal} /$ mol. Ligand protein interactions of these compounds in complex with the RNA promoter including the initiation primer and 3 '-elongation template from polio virus (model-IV) shown that both AZT-TP and T1105_TP primarily interact with PB2_N, residues near motif-II and motif-IV, and the PB1 priming loop that plays an important role in stabilizing the ligand-target interaction. In particular, $\mathrm{His}^{649}$ of the priming loop of PB1 may play an important role in stabilizing the compound within the catalytic site of the IAV RdRp. This binding orientation in turn shields $\mathrm{Asp}^{445}$ and $\mathrm{Asp}^{446}$ which are essential for polymerase activity of influenza virus within the catalytic site [53]. These two residues coordinate the metal ions involved in the catalysis. When either of these Asp-Asp residues were mutated to $\mathrm{Asp}^{445} \mathrm{His}, \mathrm{Asp}{ }^{446} \mathrm{Tyr}$, Asp ${ }^{446}$ Asn or $\mathrm{Asp}^{446} \mathrm{Glu}$, the catalytic activity of PB1 is completely abolished [54]. The Asp ${ }^{446}$ may deprotonate the 3 '-hydroxy group of the priming NTP by acting as a general base and helps the $\alpha$-phosphate of the NTP to be incorporated into the activator strand. The suitable geometry for the reaction to happen is provided by $\mathrm{His}^{649}$ of the priming loop. Mutational analysis of $\mathrm{His}^{649} \mathrm{~A}$ or $\operatorname{Pro}^{647} \mathrm{~A}$ individually, impair terminal de novo initiation on a vRNA promoter with $\mathrm{P}^{651} \mathrm{~A}$ having the strong effect [55]. To coordinate with the catalytic magnesium ion, the conformational change of $\mathrm{Asp}^{446}$ side chain with $3.5 \AA$ is required to allow the polymerase to initiate polymerization. Apart from these interactions, these compounds have the ability to disturb the structural stability of the priming loop by interacting with the nearby residues Gly ${ }^{650}$ of the PB1 central domain. However, compounds other than AZT-TP and T1105 bind at the 3 '-end of the elongation strand of vRNA and may inhibit the 
process of initiation and elongation. Based on the binding modes, it might be speculated that AZT-TP and 1105 may induce some structural changes of motifs II and IV involved in catalytic mechanism and the putative NTP tunnel formation. Mutational analysis of Lys ${ }^{481}$ of motif-IV to Gln and Arg caused a drastic reduction in the catalytic activity of PB1 ranging from 1 to $7 \%$ compared to wild-type which shows the importance of this amino acid in viral replicase transcriptase activity of PB1 [54]. The conserved residues of motif D, Lys ${ }^{480}$ and Lys ${ }^{481}$ involved in NTP binding is further stabilized by contacts with PA helix $\alpha 20$ (656-663) and the PA peptide 671-684 [4]. Thus, these compounds interfere in stacking interactions between $\mathrm{His}^{649}$ and Pro ${ }^{651}$ with the incoming NTP by binding to the catalytic site and inhibit the activity of viral polymerase. Previous mutational analysis of $\mathrm{P}^{651} \mathrm{~A}$ resulted in impairment of terminal de novo initiation of a vRNA to cRNA replication but had little effect on cRNA to vRNA replication or on cap-dependent replication [55]. This mutational effect is stronger compared to individual mutations of other two fully conserved $\left(\mathrm{H}^{649} \mathrm{~A}\right.$, $\left.\mathrm{G}^{650} \mathrm{~A}\right)$ and partially conserved amino acid $\left(\mathrm{P}^{647} \mathrm{~A}\right)$, at the tip of the PB1 $\beta$-hairpin. Further mutational analysis of $\mathrm{P}^{651} \mathrm{~F}$ showed increased activity in the formation of the trimeric complex and promoter RNA binding compared to $\mathrm{P}^{651} \mathrm{~A}$. Also, previous mutational analysis of $\mathrm{His}^{649} \mathrm{Phe}$ or $\mathrm{His}^{649} \mathrm{Arg}$ showed decreased activity compared to $\mathrm{His}^{649} \mathrm{Ala}$, while $\mathrm{His}^{649} \mathrm{Arg}$ was active as His ${ }^{649}$ Ala. However, both these mutants showed trimer formation and endonuclease activity similar to wild type RdRp [55]. These previous experimental studies which are consistent with our modelling studies showed that His ${ }^{649}$ nearby the triphosphate group of AZT-TP and T1105 might play an important role in internal initiation of vRNA. The experimental results also reveal that AZT-TP showed equally potent with 2 '-fluorodGTP used in the study with $1.12 \mu \mathrm{M}$. This may be due to inhibition of influenza virus RNA polymerase by preventing the incorporation on incoming NTPs. Based on the docking conformation, it was observed that AZT-TP interactions were mediated primarily by the thymidine and its azido group exhibiting only a modest $\mathrm{IC}_{50}$ of $1.12 \mu \mathrm{M}$ using a TCA precipitation assay. Thus, these docking studies in model-IV confirm that known influenza antiviral compounds that bind to the cavity at the 3 '-end of the vRNA activator strand or catalytic site could impair the conformational change of the priming loop necessary for the transition to elongation. Binding of the compounds at the end of the exit channel may act as chain terminators by being incorporated into the elongating nascent RNA molecule. However, compounds that bind nearer to the $\mathrm{PB} 2$ linker domain may inhibit the process of cap-snatching to generate short capped primers that initiate the synthesis of viral mRNAs. Lastly, compounds that bind nearer to the 5'-hook may disturb the passage of incoming natural NTP substrates to bind in a site very close to its catalytic position. Ligand protein interactions of these compounds show that the residues $\mathrm{Gln}^{591}, \mathrm{Ser}^{594}$, $\mathrm{Lys}^{664}$, from PA; $\mathrm{Phe}^{22}, \mathrm{Tyr}^{30}{ }^{30} \mathrm{His}^{32}, \mathrm{Asp}^{41}, \mathrm{Asn}^{61}, \mathrm{Lys}^{121}, \mathrm{Leu}^{122}$, Gly ${ }^{125}$, $\mathrm{Arg}^{126}, \mathrm{Gln}^{127}, \mathrm{Arg}^{135}, \mathrm{Asn}^{136}, \mathrm{Gln}^{137}, \mathrm{Pro}^{138}, \mathrm{Gly}^{234}, \mathrm{Lys}^{235}$, $\mathrm{Pro}^{244}, \mathrm{Arg}^{249}, \mathrm{Tyr}^{253}, \mathrm{Lys}^{279}, \mathrm{Met}^{290}, \mathrm{Arg}^{287}, \mathrm{Ala}^{280}, \mathrm{Asp}^{305}$, $\mathrm{Phe}^{412}, \mathrm{Asn}^{413}, \mathrm{Lys}^{481}, \mathrm{Thr}^{487}, \mathrm{Ser}^{494}, \mathrm{Phe}^{495}, \mathrm{Tyr}^{497}, \mathrm{Tyr}^{499}$, $\mathrm{Gly}^{500}, \mathrm{Phe}^{501}, \mathrm{Val}^{502}, \mathrm{Ala}^{503}, \mathrm{Asp}^{538}, \mathrm{Ala}^{648}, \mathrm{His}^{649}$ from PB1 and $\mathrm{Pro}^{56}, \mathrm{Arg}^{138}, \mathrm{Val}^{139}$, $\mathrm{Lys}^{140}, \mathrm{Ile}^{141}, \mathrm{Arg}^{142}, \mathrm{Thr}^{238}$, $\mathrm{Glu}^{241}, \mathrm{Ile}^{538}, \mathrm{Ile}^{539}, \mathrm{Asn}^{540}, \mathrm{Glu}^{543}, \mathrm{Asn}^{652}, \mathrm{Pro}^{654}, \mathrm{Asn}^{657}$, $\mathrm{Ass}^{659}$, $\mathrm{Leu}^{668}$, Gly ${ }^{669}$, Lys ${ }^{670}$, Asp ${ }^{671}$ from PB2 act as common pharmacophores for these compounds preventing the activity of $\mathrm{A} / \mathrm{PR} / 8 / 34 / \mathrm{H} 1 \mathrm{~N} 1$ influenza-A polymerase. In particular, these computational studies reveal new insights that known compounds bind to four different pockets above and below the priming loop. Pocket-A (PB1: $\operatorname{Arg}^{670}, \mathrm{Asn}^{671}$, $\mathrm{Arg}^{672}$; PB2: $\mathrm{Arg}^{46}, \mathrm{Trp}^{49}, \mathrm{Met}^{50}, \mathrm{Met}^{53}, \mathrm{Lys}^{54}, \mathrm{Tyr}^{55}, \mathrm{Pro}^{56}$, $\mathrm{Met}^{81}, \mathrm{Met}^{90}$ ) is close to the $3^{\prime}$-end of the activator strand, pocket-B (PB1:Asn ${ }^{276}, \mathrm{Lys}^{279}, \mathrm{Ala}^{280}, \mathrm{Ala}^{283}, \mathrm{Asn}^{284}, \mathrm{Met}^{290}$, $\mathrm{Thr}^{487}, \mathrm{Arg}^{486}, \mathrm{Gly}^{488}, \mathrm{Tyr}^{490}, \mathrm{Gly}^{500}, \mathrm{Phe}^{501}$; PB2: $\mathrm{Val}^{139}$, $\mathrm{Arg}^{142}$ ) at the end of the exit channel, pocket-C (PB2: $\mathrm{Gln}^{138}$, $\mathrm{Lys}^{140}, \mathrm{Ile}^{141}, \mathrm{Arg}^{143}, \mathrm{Phe}^{217}, \mathrm{Leu}^{218}, \mathrm{Pro}^{219}, \mathrm{Val}^{220}, \mathrm{Ala}^{221}$, $\mathrm{Gly}^{222}, \mathrm{Glu}^{241}, \mathrm{Met}^{243}, \mathrm{Arg}^{641}, \mathrm{Met}^{645}, \mathrm{Pro}^{654}, \mathrm{Asn}^{657}, \mathrm{Ile}^{529}$, $\mathrm{Thr}^{530}, \mathrm{Tyr}^{531}, \mathrm{Glu}^{538}, \mathrm{Ile}^{539}, \mathrm{Gly}^{541}, \mathrm{Asn}^{540}, \mathrm{Asn}^{657}, \mathrm{Gly}^{669}$, $\mathrm{Lys}^{670}, \mathrm{Val}^{667}$ ) near to the PB2 linker domain and pocket-D (PB1:Gly ${ }^{37}, \mathrm{Tyr}^{38}, \mathrm{Met}^{40}, \mathrm{Arg}^{45}, \mathrm{Tyr}^{355}, \mathrm{Ly}^{388}, \mathrm{Ser}^{384}, \mathrm{Thr}^{385}$, $\mathrm{Gln}^{392}$ ) above the surface of the $5^{\prime}$-hook of the template strand. The size and the shape of the predicted cavities are compatible with the binding of small-molecule inhibitors in potential allosteric sites. They could thus represent "druggable pockets". Especially, Pocket-A might be used as a starting point for structure-based drug design as a first possible approach for virtual screening. Thus, our preliminary attempts to identify novel pharmacological tools binding to the catalytic site of influenza-A virus culminated in the identification of the small molecule AZT-TP as an inhibitor of influenza viral replication displaying an $\mathrm{IC}_{50}$ of $1.12 \mu \mathrm{M}$. The results obtained also show for the first time that AZT-TP binds close to the catalytic site below the priming loop as shown in model-III and IV. This suggests that AZT-TP may bind to IAV RdRp after the translocation of the 3'-activator strand into the catalytic site using the capped primer (ModelIII) or to the catalytic site during the elongation phase of the activator strand (Model-IV) (Fig. S4H). Furthermore, binding mode analysis in model-II revealed that AZT-TP may disturb the structural stability of motif-IV by interacting with nearby residues mainly with $\mathrm{Phe}^{501}$ and $\mathrm{Val}^{502}$ of PB1 central. More likely PB1 $\mathrm{Phe}^{501}$ plays a main role in IAV RdRp inhibition in tunnel2 above the PB1 priming loop. Plausibly, the side chain of Lys ${ }^{140}$ and $\mathrm{Arg}^{664}$ create a favorable environment with a positive electrostatic potential for the binding of AZT-TP with the lesser binding affinity of $-13.9 \mathrm{Kcal} / \mathrm{mol}$ (Fig. S3H). Indeed, when the bound form of these structure is superposed with the unbound form, the 
difference in all atom positions for these compounds varied between 0.18 and $0.21 \AA$. This clearly indicates that this compound may bind either to the catalytic site with higher affinity or to the tunnel 2 site close to the PB2 linker domain with lower affinity of the polymerase. This causes a slight change in conformation from the closed to open state and might prevent the translocation of the template and product. Previously, it was also proposed that the influenza polymerase PB2 627 domain is necessary to recruit replicating vRNPs in RNA-free polymerases [56]. Also, AZT-TP as show in model-I might have the tendency to disturb the stability of motif-F3 contacting with Lys ${ }^{235}, \mathrm{Lys}^{237}$ and $\mathrm{Arg}^{239}$ involved in the NTP tunnel that leads to the tip of the putative priming loop with the moderate binding energy of $-14.6 \mathrm{Kcal} / \mathrm{mol}$ [4]. This shows that the catalytic site is wide enough to accommodate a bulky 3 '-azido group of L-AZT which could explain the observed polymerase inhibition activity. Specifically, projection of a 3 '-azido towards the highly conserved SDD motif in the catalytic site of IAV RdRP clearly indicates that 3'-substitution is highly beneficial for antiviral activity. This suggest that azido group on the five-membered ring could be used to improve either the binding affinity and/pharmacological properties of the compound. These studies further validated that catalytic site as the most druggable target for influenza A viral infections. By determining the $\mathrm{IC}_{50}$ profiles of AZT-TP tested experimentally, it was proved that this compound competitively inhibits the incorporation of GTP into viral RNA of influenza polymerase in a similar way to the positive control 2FdGTP. This is in good agreement with our docking studies showing that this compound binds around the catalytic site and prevents the activity of influenza RdRp. This also implies that AZT-TP may disturb the initiation of replication by inhibiting the incoming NTPs and may terminate viral elongation suggesting that after AZT-TP binds to the catalytic site, it inhibits the nucleophilic attack of the $3^{\prime}-\mathrm{OH}$ on the $\alpha$-phosphorous of the incoming NTP. The demonstrated inhibitory activity of this compound $\mathrm{IC}_{50}$ of $1.12 \mu \mathrm{M}$ is similar to the known influenza-inhibitory compound 2FdGTP. Our docking studies and enzymic inhibition data clearly indicate that AZT-TP inhibits RdRp polymerase activity. Thus, our work provides novel mechanistic insights into inhibition of influenza RdRp for future drug discovery.

\section{Conclusion}

In this study, we have successfully combined computational docking studies and enzymic inhibition assays to identify AZT-TP as an inhibitor of the catalytic site of IAV RdRp from the A/PR/8/34/H1N1 viral strain. A good correlation between the docking and experimental studies indicate that AZT-TP binds to the catalytic site inhibiting the incorporating of natural NTPs for replication initiation and elongation of IAV RdRp. The compound AZT-TP exhibits significant anti-viral activity inhibiting viral replication with a lower $\mathrm{IC}_{50}$ of $1.12 \mu \mathrm{M}$ compared to the previously known inhibitor 2FdGTP. This study clearly emphasizes the importance of the catalytic site below and above the priming loop for the future development of novel anti-influenza drugs.

\section{References}

1. Fodor E (2013) The RNA polymerase of influenza a virus: mechanisms of viral transcription and replication. Acta Virol 57(2):113-122

2. Resa-Infante P, Jorba N, Coloma R, Ortin J (2011) The influenza virus RNA synthesis machine: advances in its structure and function. RNA Biol 8(2):207-215

3. Hengrung N, El Omari K, Serna Martin I, Vreede FT, Cusack S, Rambo RP, Vonrhein C, Bricogne G, Stuart DI, Grimes JM, Fodor E (2015) Crystal structure of the RNA-dependent RNA polymerase from influenza $C$ virus. Nature 527(7576):114-117. https://doi.org/10.1038/nature15525

4. Pflug A, Guilligay D, Reich S, Cusack S (2014) Structure of influenza A polymerase bound to the viral RNA promoter. Nature 516(7531):355-360. https://doi.org/10.1038/nature14008

5. Reich S, Guilligay D, Pflug A, Malet H, Berger I, Crepin T, Hart D, Lunardi T, Nanao M, Ruigrok RW, Cusack S (2014) Structural insight into cap-snatching and RNA synthesis by influenza polymerase. Nature 516(7531):361-366. https://doi.org/10.1038/natur e14009

6. Appleby TC, Perry JK, Murakami E, Barauskas O, Feng J, Cho A, Fox D III, Wetmore DR, McGrath ME, Ray AS, Sofia MJ, Swaminathan S, Edwards TE (2015) Viral replication. Structural basis for RNA replication by the hepatitis $\mathrm{C}$ virus polymerase. Science 347(6223):771-775. https://doi.org/10.1126/science.1259210

7. Butcher SJ, Grimes JM, Makeyev EV, Bamford DH, Stuart DI (2001) A mechanism for initiating RNA-dependent RNA polymerization. Nature 410(6825):235-240. https://doi.org/10.1038/35065 653

8. Tao Y, Farsetta DL, Nibert ML, Harrison SC (2002) RNA synthesis in a cage-structural studies of reovirus polymerase lambda3. Cell 111(5):733-745

9. Te Velthuis AJ, Robb NC, Kapanidis AN, Fodor E (2016) The role of the priming loop in Influenza A virus RNA synthesis. Nat Microbiol 1 (5). https://doi.org/10.1038/nmicrobiol.2016.29

10. Babar MM, Zaidi NU, Tahir M (2014) Global geno-proteomic analysis reveals cross-continental sequence conservation and druggable sites among influenza virus polymerases. Antiviral Res 112:120-131. https://doi.org/10.1016/j.antiviral.2014.10.013

11. Furuta Y, Takahashi K, Shiraki K, Sakamoto K, Smee DF, Barnard DL, Gowen BB, Julander JG, Morrey JD (2009) T-705 (favipiravir) and related compounds: novel broad-spectrum inhibitors of RNA viral infections. Antiviral Res 82(3):95-102. https://doi. org/10.1016/j.antiviral.2009.02.198

12. Tisdale M, Ellis M, Klumpp K, Court S, Ford M (1995) Inhibition of influenza virus transcription by 2 '-deoxy-2'-fluoroguanosine. Antimicrob Agents Chemother 39(11):2454-2458

13. Sidwell RW, Bailey KW, Wong MH, Barnard DL, Smee DF (2005) In vitro and in vivo influenza virus-inhibitory effects of viramidine. Antiviral Res 68(1):10-17. https://doi.org/10.1016/j. antiviral.2005.06.003

14. Ghanem A, Mayer D, Chase G, Tegge W, Frank R, Kochs G, Garcia-Sastre A, Schwemmle M (2007) Peptide-mediated interference 
with influenza A virus polymerase. J Virol 81(14):7801-7804. https://doi.org/10.1128/JVI.00724-07

15. Reich S, Guilligay D, Cusack S (2017) An in vitro fluorescence based study of initiation of RNA synthesis by influenza B polymerase. Nucleic Acids Res 45(6):3353-3368. https://doi. org/10.1093/nar/gkx043

16. Tomassini J, Selnick H, Davies ME, Armstrong ME, Baldwin J, Bourgeois M, Hastings J, Hazuda D, Lewis J, McClements W et al (1994) Inhibition of cap (m7GpppXm)-dependent endonuclease of influenza virus by 4-substituted 2,4-dioxobutanoic acid compounds. Antimicrob Agents Chemother 38(12):2827-2837

17. Kowalinski E, Zubieta C, Wolkerstorfer A, Szolar OH, Ruigrok RW, Cusack S (2012) Structural analysis of specific metal chelating inhibitor binding to the endonuclease domain of influenza pH1N1 (2009) polymerase. PLoS Pathog 8(8):e1002831. https:// doi.org/10.1371/journal.ppat.1002831

18. Tomassini JE, Davies ME, Hastings JC, Lingham R, Mojena M, Raghoobar SL, Singh SB, Tkacz JS, Goetz MA (1996) A novel antiviral agent which inhibits the endonuclease of influenza viruses. Antimicrob Agents Chemother 40(5):1189-1193

19. Parkes KE, Ermert P, Fassler J, Ives J, Martin JA, Merrett JH, Obrecht D, Williams G, Klumpp K (2003) Use of a pharmacophore model to discover a new class of influenza endonuclease inhibitors. J Med Chem 46(7):1153-1164. https://doi.org/10.1021/ jm020334u

20. Byrn RA, Jones SM, Bennett HB, Bral C, Clark MP, Jacobs MD, Kwong AD, Ledeboer MW, Leeman JR, McNeil CF, Murcko MA, Nezami A, Perola E, Rijnbrand R, Saxena K, Tsai AW, Zhou Y, Charifson PS (2015) Preclinical activity of VX-787, a first-inclass, orally bioavailable inhibitor of the influenza virus polymerase PB2 subunit. Antimicrob Agents Chemother 59(3):15691582. https://doi.org/10.1128/AAC.04623-14

21. Hayden FG, Sugaya N, Hirotsu N, Lee N, de Jong MD, Hurt AC, Ishida T, Sekino H, Yamada K, Portsmouth S, Kawaguchi K, Shishido T, Arai M, Tsuchiya K, Uehara T, Watanabe A, Baloxavir Marboxil Investigators G (2018) Baloxavir Marboxil for uncomplicated influenza in adults and adolescents. N Engl J Med 379(10):913-923. https://doi.org/10.1056/NEJMoa1716197

22. Muratore G, Goracci L, Mercorelli B, Foeglein A, Digard P, Cruciani G, Palu G, Loregian A (2012) Small molecule inhibitors of influenza A and B viruses that act by disrupting subunit interactions of the viral polymerase. Proc Natl Acad Sci USA 109(16):6247-6252. https://doi.org/10.1073/pnas.1119817109

23. Obayashi E, Yoshida H, Kawai F, Shibayama N, Kawaguchi A, Nagata K, Tame JR, Park SY (2008) The structural basis for an essential subunit interaction in influenza virus RNA polymerase. Nature 454(7208):1127-1131. https://doi.org/10.1038/nature0722 5

24. Massari S, Nannetti G, Goracci L, Sancineto L, Muratore G, Sabatini S, Manfroni G, Mercorelli B, Cecchetti V, Facchini M, Palu G, Cruciani G, Loregian A, Tabarrini O (2013) Structural investigation of cycloheptathiophene-3-carboxamide derivatives targeting influenza virus polymerase assembly. J Med Chem 56(24):10118-10131. https://doi.org/10.1021/jm401560v

25. Lepri S, Nannetti G, Muratore G, Cruciani G, Ruzziconi R, Mercorelli B, Palu G, Loregian A, Goracci L (2014) Optimization of small-molecule inhibitors of influenza virus polymerase: from thiophene-3-carboxamide to polyamido scaffolds. J Med Chem 57(10):4337-4350. https://doi.org/10.1021/jm500300r

26. Loregian A, Coen DM (2006) Selective anti-cytomegalovirus compounds discovered by screening for inhibitors of subunit interactions of the viral polymerase. Chem Biol 13(2):191-200. https://doi.org/10.1016/j.chembiol.2005.12.002

27. Muratore G, Mercorelli B, Goracci L, Cruciani G, Digard P, Palu G, Loregian A (2012) Human cytomegalovirus inhibitor AL18 also possesses activity against influenza A and B viruses.
Antimicrob Agents Chemother 56(11):6009-6013. https://doi. org/10.1128/AAC.01219-12

28. Fukuoka M, Minakuchi M, Kawaguchi A, Nagata K, Kamatari YO, Kuwata K (2012) Structure-based discovery of anti-influenza virus A compounds among medicines. Biochim Biophys Acta 1820(2):90-95. https://doi.org/10.1016/j.bbagen.2011.11.003

29. Kessler U, Castagnolo D, Pagano M, Deodato D, Bernardini M, Pilger B, Ranadheera C, Botta M (2013) Discovery and synthesis of novel benzofurazan derivatives as inhibitors of influenza A virus. Bioorg Med Chem Lett 23(20):5575-5577. https://doi. org/10.1016/j.bmcl.2013.08.048

30. Li L, Chang SH, Xiang JF, Li Q, Liang HH, Tang YL, Liu YF (2012) NMR identification of anti-influenza lead compound targeting at $\mathrm{PA}(\mathrm{C})$ subunit of $\mathrm{H} 5 \mathrm{~N} 1$ polymerase. Chinese Chem Lett 23(1):89-92. https://doi.org/10.1016/j.cclet.2011.09.006

31. Nakazawa M, Kadowaki SE, Watanabe I, Kadowaki Y, Takei M, Fukuda H (2008) PA subunit of RNA polymerase as a promising target for anti-influenza virus agents. Antiviral Res 78(3):194201. https://doi.org/10.1016/j.antiviral.2007.12.010

32. Giannecchini S, Wise HM, Digard P, Clausi V, Del Poggetto E, Vesco L, Puzelli S, Donatelli I, Azzi A (2011) Packaging signals in the 5'-ends of influenza virus PA, PB1, and PB2 genes as potential targets to develop nucleic-acid based antiviral molecules. Antiviral Res 92(1):64-72. https://doi.org/10.1016/j.antiv iral.2011.06.013

33. Tado M, Abe T, Hatta T, Ishikawa M, Nakada S, Yokota T, Takaku H (2001) Inhibitory effect of modified 5'-capped short RNA fragments on influenza virus RNA polymerase gene expression. Antivir Chem Chemother 12(6):353-358. https://doi. org/10.1177/095632020101200605

34. Cianci C, Colonno RJ, Krystal M (1997) Differential effect of modified capped RNA substrates on influenza virus transcription. Virus Res 50(1):65-75

35. Sali A, Blundell TL (1993) Comparative protein modelling by satisfaction of spatial restraints. J Mol Biol 234(3):779-815. https ://doi.org/10.1006/jmbi.1993.1626

36. Altschul SF, Gish W, Miller W, Myers EW, Lipman DJ (1990) Basic local alignment search tool. J Mol Biol 215(3):403-410. https://doi.org/10.1016/S0022-2836(05)80360-2

37. Needleman SB, Wunsch CD (1970) A general method applicable to the search for similarities in the amino acid sequence of two proteins. J Mol Biol 48(3):443-453

38. Thompson JD, Higgins DG, Gibson TJ (1994) CLUSTAL W: improving the sensitivity of progressive multiple sequence alignment through sequence weighting, position-specific gap penalties and weight matrix choice. Nucleic Acids Res 22(22):4673-4680

39. Colovos C, Yeates TO (1993) Verification of protein structures: patterns of nonbonded atomic interactions. Protein Sci 2(9):15111519. https://doi.org/10.1002/pro.5560020916

40. Guex N, Peitsch MC (1997) SWISS-MODEL and the Swiss-PdbViewer: an environment for comparative protein modeling. Electrophoresis 18(15):2714-2723. https://doi.org/10.1002/elps.11501 81505

41. Liang J, Edelsbrunner H, Fu P, Sudhakar PV, Subramaniam S (1998) Analytical shape computation of macromolecules: I. Molecular area and volume through alpha shape. Proteins 33(1):1-17

42. Liang J, Edelsbrunner H, Fu P, Sudhakar PV, Subramaniam S (1998) Analytical shape computation of macromolecules: II. Inaccessible cavities in proteins. Proteins 33(1):18-29

43. Goto J, Kataoka R, Hirayama N (2004) Ph4Dock: pharmacophorebased protein-ligand docking. J Med Chem 47(27):6804-6811. https://doi.org/10.1021/jm0493818

44. Clark M, Cramer RD, Vanopdenbosch N (1989) Validation of the general-purpose tripos 5.2 force-field. J Comput Chem 10(8):9821012. https://doi.org/10.1002/jcc.540100804 
45. Mackerell AD Jr, Feig M, Brooks CL III (2004) Extending the treatment of backbone energetics in protein force fields: limitations of gas-phase quantum mechanics in reproducing protein conformational distributions in molecular dynamics simulations. J Comput Chem 25(11):1400-1415. https://doi.org/10.1002/ jcc. 20065

46. Metropolis N, Rosenbluth AW, Rosenbluth MN, Teller AH, Teller E (1953) Equation of state calculations by fast computing machines. J Chem Phys 21(6):1087-1092. https://doi. org/10.1063/1.1699114

47. Sangawa H, Komeno T, Nishikawa H, Yoshida A, Takahashi K, Nomura N, Furuta Y (2013) Mechanism of action of T-705 ribosyl triphosphate against influenza virus RNA polymerase. Antimicrob Agents Chemother 57(11):5202-5208. https://doi.org/10.1128/ AAC.00649-13

48. Barauskas O, Xing W, Aguayo E, Willkom M, Sapre A, Clarke M, Birkus G, Schultz BE, Sakowicz R, Kwon H, Feng JY (2017) Biochemical characterization of recombinant influenza A polymerase heterotrimer complex: Polymerase activity and mechanisms of action of nucleotide analogs. PLoS ONE 12(10):e0185998. https ://doi.org/10.1371/journal.pone.0185998

49. Furuta Y, Takahashi K, Kuno-Maekawa M, Sangawa H, Uehara S, Kozaki K, Nomura N, Egawa H, Shiraki K (2005) Mechanism of action of T-705 against influenza virus. Antimicrob Agents Chemother 49(3):981-986. https://doi.org/10.1128/ AAC.49.3.981-986.2005

50. Sleeman K, Mishin VP, Deyde VM, Furuta Y, Klimov AI, Gubareva LV (2010) In vitro antiviral activity of favipiravir (T-705) against drug-resistant influenza and 2009 A(H1N1) viruses. Antimicrob Agents Chemother 54(6):2517-2524. https ://doi.org/10.1128/AAC.01739-09

51. Graci JD, Cameron CE (2006) Mechanisms of action of ribavirin against distinct viruses. Rev Med Virol 16(1):37-48. https://doi. org/10.1002/rmv.483

52. Cheung PP, Watson SJ, Choy KT, Fun Sia S, Wong DD, Poon LL, Kellam P, Guan Y, Malik Peiris JS, Yen HL (2014) Generation and characterization of influenza A viruses with altered polymerase fidelity. Nat Commun 5:4794. https://doi.org/10.1038/ncomm s5794

53. Zamyatkin DF, Parra F, Alonso JM, Harki DA, Peterson BR, Grochulski P, Ng KK (2008) Structural insights into mechanisms of catalysis and inhibition in Norwalk virus polymerase. J Biol Chem 283(12):7705-7712. https://doi.org/10.1074/jbc.M709563200

54. Biswas SK, Nayak DP (1994) Mutational analysis of the conserved motifs of influenza A virus polymerase basic protein 1 . J Virol 68(3): 1819-1826

55. Te Velthuis AJ, Robb NC, Kapanidis AN, Fodor E (2016) The role of the priming loop in influenza A virus RNA synthesis. Nat Microbiol 1:16029. https://doi.org/10.1038/nmicrobiol.2016.29

56. Nilsson BE, Te Velthuis AJ, Fodor E (2017) Role of the PB2 627 domain in influenza A virus polymerase function. J Virol. https:// doi.org/10.1128/JVI.02467-16

Publisher's Note Springer Nature remains neutral with regard to jurisdictional claims in published maps and institutional affiliations. 